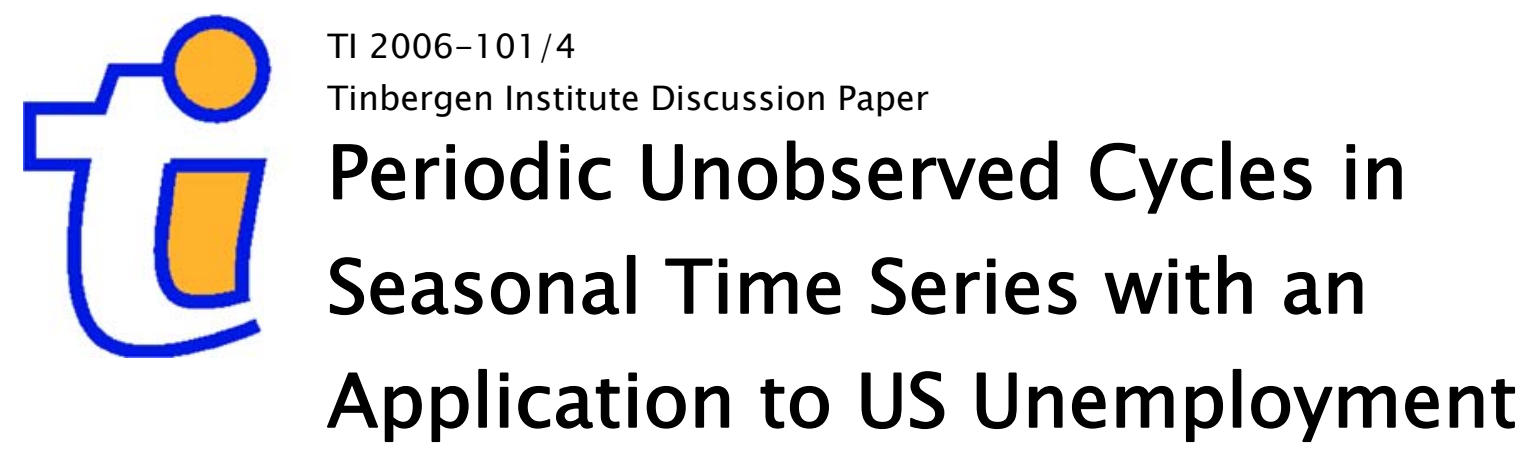

Siem Jan Koopman ${ }^{1,2}$

Marius Ooms ${ }^{\prime}$

Irma Hindrayanto ${ }^{1,2}$

${ }^{\prime}$ Faculty of Economics and Business Administration, Vrije Universiteit Amsterdam,

2 Tinbergen Institute. 


\section{Tinbergen Institute}

The Tinbergen Institute is the institute for economic research of the Erasmus Universiteit Rotterdam, Universiteit van Amsterdam, and Vrije Universiteit Amsterdam.

Tinbergen Institute Amsterdam

Roetersstraat 31

1018 WB Amsterdam

The Netherlands

Tel.: $\quad+31(0) 205513500$

Fax: $\quad+31(0) 205513555$

Tinbergen Institute Rotterdam

Burg. Oudlaan 50

3062 PA Rotterdam

The Netherlands

Tel.: $\quad+31(0) 104088900$

Fax: $\quad+31(0) 104089031$

Most TI discussion papers can be downloaded at http:/ /www.tinbergen.nl. 


\title{
Periodic unobserved cycles in seasonal time series with an application to US unemployment
}

\author{
Siem Jan Koopman*, Marius Ooms, Irma Hindrayanto \\ Vrije Universiteit Amsterdam \\ Tinbergen Institute Amsterdam
}

\begin{abstract}
This paper discusses identification, specification, estimation and forecasting for a general class of periodic unobserved components time series models with stochastic trend, seasonal and cycle components. Convenient state space formulations are introduced for exact maximum likelihood estimation, component estimation and forecasting. Identification issues are considered and a novel periodic version of the stochastic cycle component is presented. In the empirical illustration, the model is applied to postwar monthly US unemployment series and we discover a significantly periodic cycle. Furthermore, a comparison is made between the performance of the periodic unobserved components time series model and a periodic seasonal autoregressive integrated moving average model. Moreover, we introduce a new method to estimate the latter model.
\end{abstract}

Key words: Unobserved component models, state space methods, seasonal adjustment, time-varying parameters, forecasting.

JEL classification: C22, C51, E32, E37.

\footnotetext{
${ }^{*}$ Corresponding author: S.J. Koopman, Department of Econometrics, Vrije Universiteit Amsterdam, De Boelelaan 1105, 1081 HV Amsterdam, The Netherlands. Phone: +31 2059860 19; e-mail: s.j.koopman@feweb.vu.nl
} 


\section{Introduction}

Seasonal time series with sample autocorrelation functions that change with the season are referred to as periodic time series. To enable the identification of such dynamic characteristics in a time series, Tiao \& Grupe (1980) have formally defined periodic autocorrelations. Once periodic properties of a time series are detected, the time series analyst can consider time series models that allow for these periodic correlations. A model-based periodic time series analysis becomes effective when appropriate methods and algorithms are developed for estimation, decomposition, diagnostic checking and forecasting. This aim is the primary concern of our paper.

The dynamic properties of a particular seasonal time series model are governed by parameters that are usually assumed fixed throughout a given time period. For example, in the context of autoregressive moving average (ARMA) models, the parameters associated with the AR and MA lag polynomials are oftentimes assumed fixed. In case these parameters are allowed to be deterministic functions of the season index, the ARMA model becomes part of the class of periodic linear time series models. Various developments on periodic time series are given in the statistics and econometrics literature. Furthermore, many environmental and economic studies have given empirical evidence that time series models require periodically changing parameters, see for example, Bloomfield, Hurd \& Lund (1994) and Ooms and Franses (1997, 2001).

Periodic models have been introduced as early as 1955 in the article of Hannan (1955) and found widespread interest in geophysics and environmental empirical studies. The notable vector representation of periodic time series was proposed by Gladysev (1961). Osborn \& Smith (1989) introduced the periodic framework in dynamic macroeconomic models while Franses \& Paap (2004) discussed periodic analyses and applications in econometrics. In the context of autogressive models, Franses, Boswijk \& Haldrup (1997) derived tests for periodic stochastic nonstationarity and Burridge \& Taylor (2004) developed simulation based seasonal unit root tests in the presence of periodic heteroskedasticity. Exact maximum likelihood estimation methods for ARMA models are discussed by Jimenez, McLeod \& Hipel (1989) and Lund \& Basawa (2000). Anderson \& Meerschaert (2005) provide asymptotic theory for efficient moment based estimation. Most of these periodic studies explore periodic versions of ARMA models. Economic applications are often limited to quarterly data. In hydrology and meteorology there are more high frequency applications, see, i.a., the study by Lund, Shao \& Basawa (2006) where a Fourier parameterisation of periodic ARMA models is applied to a daily time series of temperature data and Tesfaye, Meerschaert \& Anderson (2006) for periodic ARMA modelling of monthly river flows. 
In this paper we focus on periodic extensions of the unobserved components (UC) time series models that are reviewed in Harvey (1989). This set of linear models describes a time series process as a sum of components that can be directly interpreted as trend, season and cycle. Each component is specified as an independent linear dynamic process. For example, the trend component can be associated with a random walk process while the cycle component can be modelled as a stationary ARMA process. Additional to lag polynomial coefficients, parameters consist of variances associated with disturbances that drive the random walk and ARMA processes. Once these parameters are allowed to be deterministic functions of the season index, the resulting UC models are referred to as periodic unobserved components time series models. Earlier periodic extensions of the UC models have been explored by Koopman and Ooms (2002, 2003, 2006), Proietti (2004) and Tripodis \& Penzer (2004). In these contributions, straightforward applications of the vector representation of periodic UC models are considered or only specific parameters in the model are considered to be periodic. More specifically, Proietti (2004) considers a UC model with the trend component modelled as a weighted average of separate independent random walks for each season, Tripodis \& Penzer (2004) consider a seasonal component with a periodic variance, while Koopman and Ooms $(2002,2006)$ explore different periodic specifications of the UC model using standard available software.

We present a comprehensive treatment of a general class of periodic UC models that include trend, seasonal and stationary cyclical components. Given the general concepts of both UC and periodic models, there are many ways to specify a periodic UC model. In case the periodic UC model is represented as a vector of independent time series where each element represents a particular season, the seasonal component is effectively eliminated. The seasonal process can not be identified from an observed time series when remaining components are periodically independent for all seasons. Since we are particularly concerned with the decomposition of a time series into trend, seasonal and cycle, we propose a convenient periodic formulation of the UC model that preserves the ability to extract a seasonal component from a time series (seasonal adjustment). These periodic UC models can still be represented in the Gladysev vector form but the components do not consist of periodically independent processes. Each component remains linearly dependent of a common underlying stochastic process for all periods. As a result, this approach facilitates the detrending, seasonal adjustment and trend-cycle decomposition of a time series based on a periodic model.

Since all parameters of the model, except for the cycle lengths, will be considered as periodic, the number of parameters in a periodic UC model increases rapidly with the seasonal length. Therefore, a specific attention is given to the identification of the parameters in the model. 
Perhaps somewhat counter-intuitive, it turns out that some parameters can not be identified when the seasonal length $S$ is small, particularly for a bi-annual time series where $S=2$. However, when the seasonal length is sufficiently large, all parameters can be identified.

Many aspects of time series analysis will be discussed and implemented for this class of periodic UC models, including signal extraction, seasonal adjustment, trend-cycle decomposition, diagnostic checking of standardised prediction errors and forecasting. In the empirical illustration, we apply the periodic model to a long monthly time series of postwar US unemployment. The unemployment series is chosen as it is a key variable in economics. More importantly, it is also subject to seasonal variation since labour supply and demand in many sectors of the economy depend on seasonal factors such as school calendars, summer work, winter breaks, et cetera. Furthermore, it is well known that unemployment is highly dependent on business cycle dynamics and therefore cycle components also need to be considered in the analysis. Most important for this paper, it is argued that unemployment is also subject to significant periodic serial correlation. The empirical results obtained by the periodic UC model will be compared with those of a periodic seasonal ARMA model. Estimation procedures for all periodic models are based on exact maximum likelihood procedures using computationally efficient state space methods. The approach taken is also novel in the context of estimating parameters in a periodic seasonal autoregressive integrated moving average (SARIMA) model.

The remaining part of the paper is organised as follows. The next section reviews the UC model. The third section introduces periodic UC models and corresponding univariate and multivariate state space representations. It also addresses asymptotic identification by considering systems of moment conditions. In the last part of this section a novel periodic version of the stochastic cycle model is presented. The fourth section analyses monthly US unemployment using both periodic SARIMA and periodic UC models where we reveal significant periodicity in the cycle component. We also take account of the cycle variance moderation in postwar US unemployment in our models. The fifth section concludes. The Appendices discuss extensions and details.

\section{Unobserved component models}

In this section we introduce unobserved component (UC) time series models. The established notation following Harvey (1989) and Durbin \& Koopman (2001) will be used throughout the paper. The univariate unobserved components time series model that is particularly suitable for many economic data sets is given by

$$
y_{t}=\mu_{t}+\gamma_{t}+\psi_{t}+\varepsilon_{t}, \quad \varepsilon_{t} \sim \operatorname{NID}\left(0, \sigma_{\varepsilon}^{2}\right), \quad t=1, \ldots, n,
$$


where $\mu_{t}, \gamma_{t}, \psi_{t}$ and $\varepsilon_{t}$ represent trend, seasonal, cyclical and irregular components, respectively. The trend and seasonal components are modelled by linear dynamic stochastic processes driven by random disturbances. The cycle is based on a stochastic trigonometric function that relies on a damping factor, frequency and random disturbances.

The simplest form of a UC model, the so-called local level model, is obtained from equation (1) where $\gamma_{t}$ and $\psi_{t}$ are zero for all $t$. The trend can be specified as the random walk process

$$
\mu_{t+1}=\mu_{t}+\eta_{t}, \quad \eta_{t} \sim \operatorname{NID}\left(0, \sigma_{\eta}^{2}\right)
$$

for $t=1, \ldots, n$. By adding a slope term $\beta_{t}$, that is also generated by a random walk to equation (2), we obtain the so-called local linear trend model,

$$
\begin{array}{lll}
\mu_{t+1}=\mu_{t}+\beta_{t}+\eta_{t}, & \eta_{t} \sim \operatorname{NID}\left(0, \sigma_{\eta}^{2}\right), \\
\beta_{t+1}=\beta_{t}+\zeta_{t}, & \zeta_{t} \sim \operatorname{NID}\left(0, \sigma_{\zeta}^{2}\right),
\end{array}
$$

for $t=1, \ldots, n$, where the trend and slope disturbances, $\eta_{t}$ and $\zeta_{t}$, respectively, are mutually uncorrelated sequences from a Gaussian density with zero mean and variance $\sigma_{\eta}^{2}$ for $\eta_{t}$ and $\sigma_{\zeta}^{2}$ for $\zeta_{t}$. If $\sigma_{\zeta}^{2}$ is zero, we have $\zeta_{t}=0$ and $\beta_{t+1}=\beta_{t}=\beta$ for all $t$. This implies a random walk plus drift process for the trend $\mu_{t}$. When $\sigma_{\eta}^{2}$ is zero as well, a deterministic linear trend is obtained for $\mu_{t}$. If only $\sigma_{\eta}^{2}$ is zero, then we have a so-called smooth trend model or an integrated random walk process. This implies that $\Delta \mu_{t}$ follows a random walk process where $\Delta=1-L$ is the difference operator and $L$ is the lag operator with $L^{p} y_{t}=y_{t-p}$.

To take account of the seasonal variation in the time series $y_{t}$, the seasonal component $\gamma_{t}$ is included. A deterministic seasonal component should have the property that it sums to zero over the previous year to ensure that it is not confounded with the trend. Flexibility of the seasonal component is achieved when it is allowed to change over time. This can be established by adding a disturbance term (with mean zero) to the sum of the $S$ seasonal effects over the past year. In this way we obtain the stochastic dummy variable form of the seasonal component as given by

$$
S_{S}(L) \gamma_{t+1}=\omega_{t}, \quad \omega_{t} \sim \operatorname{NID}\left(0, \sigma_{\omega}^{2}\right)
$$

where $S_{S}(L)$ is the summation operator defined as $S_{S}(L)=1+L+\cdots+L^{S-1}$. Since economic time series are often subject to cyclical dynamics, a stochastic cycle may be included in the model and be specified as

$$
\left(\begin{array}{c}
\psi_{t+1} \\
\psi_{t+1}^{*}
\end{array}\right)=\rho\left(\begin{array}{cc}
\cos \lambda & \sin \lambda \\
-\sin \lambda & \cos \lambda
\end{array}\right)\left(\begin{array}{c}
\psi_{t} \\
\psi_{t}^{*}
\end{array}\right)+\left(\begin{array}{c}
\kappa_{t} \\
\kappa_{t}^{*}
\end{array}\right), \quad 0<\rho<1,
$$


where the period of the cycle is given by $2 \pi / \lambda$. This dynamic process can be written as an ARMA process with complex roots in the autoregressive polynomial. It therefore generates a cyclical pattern in the theoretical autocorrelation function of process (5).

\section{Periodic unobserved components models}

Univariate seasonal time series $y_{t}$ are considered with seasonal length $S$ ( $S=4$ for quarterly data and $S=12$ for monthly data). Seasonal time series are often analysed by seasonal autoregressive moving average (SARMA) models and by the UC models of the previous section. The standard formulations of these models assume that all parameters are constant through time. In case the models are periodic, the parameters are allowed to vary with the season. As a result, the number of parameters increases by a multiple of $S$. This section develops a statistical periodic time series approach for univariate unobserved component models with trend, season, cycle and irregular.

We start our analysis in $\S 3.1$ with the simplest periodic basic structural model (BSM) which contains only three components: trend, season and irregular. Considering the moments of this model, we show that the univariate time-varying-parameter BSM does not correspond to a multivariate constant-parameter BSM. Next, in $\S 3.2$ we develop two convenient ways of putting a periodic UC model into state space form, namely a univariate time-varying and a multivariate time-invariant representation. Both forms enable exact maximum likelihood (ML) estimation, filtering and smoothing. In $\S 3.3$ we derive the second order moments of the periodic BSM and we argue that not all the parameters of periodic UC models are automatically identified. Section 3.4 introduces a novel periodic stochastic cycle component. We derive the moments and we show that exact ML estimation can be implemented without additional identifying restrictions.

\subsection{Periodic basic structural time series model}

Consider a univariate basic structural time series model (BSM) with periodic variances for the disturbances associated with trend, seasonal and irregular components. This model can be expressed by

$$
\begin{array}{rlrl}
y_{t} & =\mu_{t}+\gamma_{t}+\varepsilon_{t}, & & \varepsilon_{t} \sim \operatorname{NID}\left(0, \sigma_{\varepsilon, s}^{2}\right), \\
\mu_{t+1}=\mu_{t}+\eta_{t}, & \eta_{t} \sim \operatorname{NID}\left(0, \sigma_{\eta, s}^{2}\right), \\
\gamma_{t+1}=-\sum_{j=0}^{S-2} \gamma_{t-j}+\omega_{t}, & \omega_{t} \sim \operatorname{NID}\left(0, \sigma_{\omega, s}^{2}\right),
\end{array}
$$

for $t=1, \ldots, n, n=n^{*} S$, where $\sigma_{\varepsilon, s}^{2}, \sigma_{\eta, s}^{2}$ and $\sigma_{\omega, s}^{2}$ are the variances for $\varepsilon_{t}, \eta_{t}$ and $\omega_{t}$ respectively, and for season $s=1, \ldots, S$. To simplify notation for the multivariate representations of the 
model, we assume we have $n^{*}$ complete years of data, but this assumption is not essential for the subsequent statistical analysis.

Once model (6) is written as a multivariate process (each equation is for a particular season), it is shown below that the periodic BSM (6) does not reduce to a standard multivariate local level model for yearly observations. Denote $y_{s, t^{*}}$ as the observation for period $s$ and year $t^{*}$ such that $y_{t} \equiv y_{s, t^{*}}$, where $t=(S-1) t^{*}+s$ for $t=1, \ldots, n^{*} S, t^{*}=1, \ldots, n^{*}$ and $s=1,2, \ldots, S$. For the case of $S=2$ we have

$$
y_{1, t^{*}}=\mu_{1, t^{*}}+\varepsilon_{1, t^{*}}, \quad y_{2, t^{*}}=\mu_{2, t^{*}}+\varepsilon_{2, t^{*}}
$$

where

$$
\mu_{1, t^{*}}=\mu_{t}+\gamma_{t}, \quad \mu_{2, t^{*}}=\mu_{t+1}+\gamma_{t+1}, \quad \varepsilon_{1, t^{*}}=\varepsilon_{t}, \quad \varepsilon_{2, t^{*}}=\varepsilon_{t+1},
$$

so that the trend becomes

$$
\mu_{1, t^{*}+1}=\mu_{1, t^{*}}+\eta_{1, t^{*}}, \quad \mu_{2, t^{*}+1}=\mu_{2, t^{*}}+\eta_{2, t^{*}}
$$

The trend disturbances in (9) include the seasonal disturbances by construction, since it follows that

$$
\eta_{1, t^{*}}=\eta_{t}+\eta_{t+1}-\omega_{t}+\omega_{t+1}, \quad \eta_{2, t^{*}}=\eta_{t+1}+\eta_{t+2}-\omega_{t+1}+\omega_{t+2}
$$

In matrix form, the above model can be written as

$$
\begin{array}{r}
y_{t^{*}}^{*}=\mu_{t^{*}}^{*}+\varepsilon_{t^{*}}^{*} \\
\mu_{t^{*}+1}^{*}=\mu_{t^{*}}^{*}+\eta_{t^{*}}^{*}
\end{array}
$$

where we denote the vectors as $y_{t^{*}}^{*}=\left(y_{1, t^{*}}, y_{2, t^{*}}\right)^{\prime}, \mu_{t^{*}}^{*}=\left(\mu_{1, t^{*}}, \mu_{2, t^{*}}\right)^{\prime}, \varepsilon_{t^{*}}^{*}=\left(\varepsilon_{1, t^{*}}, \varepsilon_{2, t^{*}}\right)^{\prime}$ and $\eta_{t^{*}}^{*}=\left(\eta_{1, t^{*}}, \eta_{2, t^{*}}\right)^{\prime}$. The variance matrix of the total disturbance vector $\left(\varepsilon_{1, t^{*}}, \varepsilon_{2, t^{*}}, \eta_{1, t^{*}}, \eta_{2, t^{*}}\right)^{\prime}$ is given by

$$
\left(\begin{array}{cccc}
\sigma_{\varepsilon, 1}^{2} & 0 & 0 & 0 \\
0 & \sigma_{\varepsilon, 2}^{2} & 0 & 0 \\
0 & 0 & \sigma_{\eta, 1}^{2}+\sigma_{\eta, 2}^{2}+\sigma_{\omega, 1}^{2}+\sigma_{\omega, 2}^{2} & \sigma_{\eta, 2}^{2}-\sigma_{\omega, 2}^{2} \\
0 & 0 & \sigma_{\eta, 2}^{2}-\sigma_{\omega, 2}^{2} & \sigma_{\eta, 1}^{2}+\sigma_{\eta, 2}^{2}+\sigma_{\omega, 1}^{2}+\sigma_{\omega, 2}^{2}
\end{array}\right)
$$

Further,

$$
\mathbb{E}\left[\left(\begin{array}{l}
\eta_{1, t^{*}+1} \\
\eta_{2, t^{*}+1}
\end{array}\right)\left(\begin{array}{l}
\eta_{1, t^{*}} \\
\eta_{2, t^{*}}
\end{array}\right)^{\prime}\right]=\left(\begin{array}{cc}
0 & \sigma_{\eta, 1}^{2}-\sigma_{\omega, 1}^{2} \\
0 & 0
\end{array}\right)
$$


and

$$
\mathbb{E}\left[\left(\begin{array}{c}
\eta_{1, t^{*}+j} \\
\eta_{2, t^{*}+j}
\end{array}\right)\left(\begin{array}{l}
\eta_{1, t^{*}} \\
\eta_{2, t^{*}}
\end{array}\right)^{\prime}\right]=0_{2 \times 2} \quad \text { for } j>1
$$

Since (14) is not a zero matrix, the level disturbance vector $\eta_{t^{*}}^{*}$ follows a moving average process. Therefore, we do not obtain a standard multivariate version of the local level model with a serially independent sequence of $\eta_{t^{*}}^{*}$. Unfortunately, it means we cannot estimate this simple model using standard software for multivariate basic structural models. This is a disadvantage compared with other periodic unobserved component models discussed in Koopman \& Ooms (2006). To implement maximum likelihood estimation, filtering and smoothing we therefore have to develop different convenient state space forms.

\subsection{Convenient state space representations of the periodic BSM}

The state space form provides a unified representation of a wide range of linear Gaussian time series models including the structural time series model; see, for example, Harvey (1989), Kitagawa \& Gersch (1996) and Durbin \& Koopman (2001). The Gaussian state space form consists of a transition equation for the $m \times 1$ vector $\alpha_{t}$ and a measurement equation for the $N \times 1$ vector $y_{t}$. We formulate the model as in de Jong (1991), that is

$$
\begin{aligned}
& \alpha_{t+1}=T_{t} \alpha_{t}+H_{t} \epsilon_{t}, \quad \alpha_{1} \sim \mathcal{N}\left(a, P_{0}\right), \quad t=1, \ldots, n, \\
& y_{t}=Z_{t} \alpha_{t}+G_{t} \epsilon_{t}, \quad \epsilon_{t} \sim \operatorname{NID}(0, I),
\end{aligned}
$$

where $\epsilon_{t}$ is an independent sequence of standard normal distributed random vectors. The matrices $T_{t}, H_{t}, Z_{t}$ and $G_{t}$ are referred to as the state space system matrices. The observations at time $t$ are placed in an $N \times 1$ vector $y_{t}$ for $t=1, \ldots, n$. Periodic models can be formulated with a univariate measurement equation and time varying system matrices, $N=1, T_{t}, H_{t}, Z_{t}$ and $G_{t}$, $t=1, \ldots, n$ or with a multivariate measurement equation for $y_{t^{*}}^{*}$ and constant system matrices, where $N=S$ equal to the number of seasons per year. We discuss two convenient state space representations, (16)-(17) of (6), one univariate, and one multivariate.

The state space matrices of the univariate time-varying-parameter form of (6) are given by

$$
\begin{gathered}
T=\left[\begin{array}{cc}
1 & 0 \\
0 & -1
\end{array}\right], \quad H_{t}=\left[\begin{array}{ccc}
0 & \sigma_{\eta, t} & 0 \\
0 & 0 & \sigma_{\omega, t}
\end{array}\right], \\
Z=\left[\begin{array}{ll}
1 & 1
\end{array}\right], \quad G_{t}=\left[\begin{array}{lll}
\sigma_{\varepsilon, t} & 0 & 0
\end{array}\right],
\end{gathered}
$$


where $\sigma_{\eta, t}, \sigma_{\omega, t}$ and $\sigma_{\eta, t}$ vary deterministically according to the season. Note that the matrices $H_{t}$ and $G_{t}$ are time-varying, while $T$ and $Z$ are constant over time. The state vector $\alpha_{t}=\left(\begin{array}{ll}\mu_{t} & \gamma_{t}\end{array}\right)^{\prime}$ and $\epsilon_{t}$ is a $3 \times 1$ vector. The initialization of $\alpha_{t}$ is diffuse: $a_{1}$ is a vector of 0 's and $P_{0}$ is $\kappa I_{2}$ with $\kappa \rightarrow \infty$.

The multivariate time-invariant state space form of model (6) can be written as:

$$
\begin{aligned}
\alpha_{t^{*}+1}^{*} & =T^{*} \alpha_{t^{*}}^{*}+H^{*} \epsilon_{t^{*}}^{*}, & & \alpha_{1}^{*} \sim \mathcal{N}\left(a^{*}, P_{0}^{*}\right), \\
y_{t^{*}}^{*} & =Z^{*} \alpha_{t^{*}}^{*}+G^{*} \epsilon_{t^{*}}^{*}, & \epsilon_{t^{*}}^{*} & \sim \operatorname{NID}(0, I) .
\end{aligned}
$$

To simplify notation we consider model (6) for $S=2$, where $y_{t^{*}}^{*}=\left(y_{t}, y_{t+1}\right)^{\prime}, t=1,1+S, 1+$ $2 S, \ldots, 1+\left(n^{*}-1\right) S$. We derive convenient expressions for $\alpha_{t^{*}}^{*}, \epsilon_{t^{*}}^{*}, T^{*}, H^{*}, Z^{*}$ and $G^{*}$ as follows. The measurement equations are given by

$$
\begin{array}{rlrl}
y_{t} & =\mu_{t}+\gamma_{t}+\varepsilon_{t}, & \varepsilon_{t} & \sim \operatorname{NID}\left(0, \sigma_{\varepsilon, 1}^{2}\right), \\
y_{t+1}=\mu_{t+1}+\gamma_{t+1}+\varepsilon_{t+1}=\mu_{t}+\eta_{t}-\gamma_{t}+\omega_{t}+\varepsilon_{t+1}, & & \varepsilon_{t+1} \sim \operatorname{NID}\left(0, \sigma_{\varepsilon, 2}^{2}\right) .
\end{array}
$$

Further, we take $\alpha_{t^{*}}^{*}=\left(\mu_{t}, \eta_{t}, \gamma_{t}, \omega_{t}\right)^{\prime}$ as the state vector and it follows from the transition equations that

$$
\begin{array}{ll}
\mu_{t+2}=\mu_{t+1}+\eta_{t+1}=\mu_{t}+\eta_{t}+\eta_{t+1}, & \eta_{t+1} \sim \operatorname{NID}\left(0, \sigma_{\eta, 2}^{2}\right), \\
\gamma_{t+2}=-\gamma_{t+1}+\omega_{t+1}=\gamma_{t}-\omega_{t}+\omega_{t+1} & \omega_{t+1} \sim \operatorname{NID}\left(0, \sigma_{\omega, 2}^{2}\right) .
\end{array}
$$

The state space matrices are then given by

$$
\begin{aligned}
& T^{*}=\left(\begin{array}{cccc}
1 & 1 & 0 & 0 \\
0 & 0 & 0 & 0 \\
0 & 0 & 1 & -1 \\
0 & 0 & 0 & 0
\end{array}\right), \quad H^{*}=\left(\begin{array}{cccccc}
0 & 0 & \sigma_{\eta, 2} & 0 & 0 & 0 \\
0 & 0 & 0 & \sigma_{\eta, 1} & 0 & 0 \\
0 & 0 & 0 & 0 & \sigma_{\omega, 2} & 0 \\
0 & 0 & 0 & 0 & 0 & \sigma_{\omega, 1}
\end{array}\right) \text {, } \\
& Z^{*}=\left(\begin{array}{cccc}
1 & 0 & 1 & 0 \\
1 & 1 & -1 & 1
\end{array}\right), \quad G^{*}=\left(\begin{array}{cccccc}
\sigma_{\varepsilon, 1} & 0 & 0 & 0 & 0 & 0 \\
0 & \sigma_{\varepsilon, 2} & 0 & 0 & 0 & 0
\end{array}\right)
\end{aligned}
$$

with a $3 S \times 1$ vector $\epsilon_{t^{*}}^{*}$, for $t^{*}=1,2, \ldots, n^{*}$ and $t=1,1+S, 1+2 S, \ldots, 1+\left(n^{*}-1\right) S$, so that $\alpha_{t^{*}+1}^{*}=\left(\mu_{t+2}, \eta_{t+2}, \gamma_{t+2}, \omega_{t+2}\right)^{\prime}$. The initialization of $\alpha_{t^{*}}^{*}$ is diffuse: $a_{1}^{*}$ is a vector of 0 's and $P_{0}$ is $\kappa I_{2 S}$ with $\kappa \rightarrow \infty$.

It is numerically verified that the multivariate time-invariant system is observationally equivalent to the univariate time varying system. In particular, the Gaussian likelihood of model (22)-(23) is exactly equal to the likelihood of model (18)-(19). Both state space representations have serially independent disturbances for the trend and seasonal component of model (6). The two 
state space forms are therefore easier to handle and interpret than the multivariate representation (11)-(12), where the trend and the seasonal component are confounded.

For both specifications, it is clear that there is only one trend for the whole observed series. Although the multivariate representation may suggest that we have a separate trend for each season, the state vector $\alpha_{t^{*}}^{*}$ only has a single trend, $\mu_{t}$, and a single seasonal component, $\gamma_{t}$. The merit of the univariate specification is its straightforward treatment although the state space formulation has time-varying system matrices. The merit of the multivariate form is that the system matrices are time-invariant, which is the standard format to examine the dynamic properties of the time series.

\subsection{Moments, identification and estimation,}

To investigate whether all parameters in the UC models are identified, we use systems of moment conditions. The moment conditions are based on the autocovariance function of the stationary form of the UC models. Consider model (6) with $S=2$ where we have 6 parameters to estimate, namely $\sigma_{\varepsilon, 1}, \sigma_{\varepsilon, 2}, \sigma_{\eta, 1}, \sigma_{\eta, 2}, \sigma_{\omega, 1}$ and $\sigma_{\omega, 2}$. The stationary form of model (6) is based on yearly differences $\Delta_{S} y_{t}=\left(1-L^{S}\right) y_{t}$ of the observations $y_{t}$. For $S=2$, that is

$$
\Delta_{2} y_{t+i}=\eta_{t+i-2}+\eta_{t+i-1}-\omega_{t+i-2}+\omega_{t+i-1}+\varepsilon_{t+i}-\varepsilon_{t+i-2}
$$

for $i=\ldots,-2,-1,0,1,2, \ldots$ and $t=1, S+1,2 S+1, \ldots$ It follows that $\mathbb{E}\left[\Delta_{2} y_{t+i}\right]=0$ for all $i$. The yearly autocovariance function for $t=1, S+1,2 S+1, \ldots$ is given by

$$
\begin{aligned}
& \Gamma_{0}=\mathbb{E}\left[\left(\begin{array}{c}
\Delta_{2} y_{t} \\
\Delta_{2} y_{t+1}
\end{array}\right)\left(\begin{array}{c}
\Delta_{2} y_{t} \\
\Delta_{2} y_{t+1}
\end{array}\right)^{\prime}\right] \\
& =\left(\begin{array}{cc}
\sigma_{\eta, 1}^{2}+\sigma_{\eta, 2}^{2}+\sigma_{\omega, 1}^{2}+\sigma_{\omega, 2}^{2}+2 \sigma_{\varepsilon, 1}^{2} & \sigma_{\eta, 2}^{2}-\sigma_{\omega, 2}^{2} \\
\sigma_{\eta, 2}^{2}-\sigma_{\omega, 2}^{2} & \sigma_{\eta, 1}^{2}+\sigma_{\eta, 2}^{2}+\sigma_{\omega, 1}^{2}+\sigma_{\omega, 2}^{2}+2 \sigma_{\varepsilon, 2}^{2}
\end{array}\right) \\
& \Gamma_{1}=\mathbb{E}\left[\left(\begin{array}{c}
\Delta_{2} y_{t} \\
\Delta_{2} y_{t+1}
\end{array}\right)\left(\begin{array}{c}
\Delta_{2} y_{t-2} \\
\Delta_{2} y_{t-1}
\end{array}\right)^{\prime}\right]=\left(\begin{array}{cc}
-\sigma_{\varepsilon, 1}^{2} & \sigma_{\eta, 1}^{2}-\sigma_{\omega, 1}^{2} \\
0 & -\sigma_{\varepsilon, 2}^{2}
\end{array}\right) \\
& \Gamma_{j}=\mathbb{E}\left[\left(\begin{array}{c}
\Delta_{2} y_{t} \\
\Delta_{2} y_{t+1}
\end{array}\right)\left(\begin{array}{c}
\Delta_{2} y_{t-2 j} \\
\Delta_{2} y_{t+1-2 j}
\end{array}\right)^{\prime}\right]=0 \quad \text { for } j=2,3,4, \ldots,
\end{aligned}
$$

which is equivalent to the autocovariance function of a vector moving average process with one lag. Note that the autocovariance matrix of lag $1, \Gamma_{1}$, is not symmetric, namely

$$
\Gamma_{-1}=\mathbb{E}\left[\left(\begin{array}{c}
\Delta_{2} y_{t} \\
\Delta_{2} y_{t+1}
\end{array}\right)\left(\begin{array}{c}
\Delta_{2} y_{t+2} \\
\Delta_{2} y_{t+3}
\end{array}\right)^{\prime}\right]=\left(\begin{array}{cc}
-\sigma_{\varepsilon, 1}^{2} & 0 \\
\sigma_{\eta, 1}^{2}-\sigma_{\omega, 1}^{2} & -\sigma_{\varepsilon, 2}^{2}
\end{array}\right)=\Gamma_{1}^{\prime} .
$$


These moment expressions reconfirm that we do not have a standard multivariate local level model for $y_{t^{*}}^{*}$ as we saw earlier in the discussion of (11)-(12).

To identify the parameters from the autocovariances, we need to solve a linear system of moment equations. Asymptotically, the expressions for the autocovariances determine the Gaussian likelihood that we use in estimation. If two instances of a time series model with different parameters have the same autocovariance function and therefore the same spectrum and the same moving average representation, the parameters cannot be identified by the Gaussian ML estimator, see, e.g. Brockwell \& Davis (1993, § 10.8) and Yao \& Brockwell (2006).

Rewriting expressions (25)-(27) we get the following system of moment equations for model (6)

$$
\left(\begin{array}{cccccc}
1 & 1 & 1 & 1 & 2 & 0 \\
0 & 1 & 0 & -1 & 0 & 0 \\
1 & 1 & 1 & 1 & 0 & 2 \\
0 & 0 & 0 & 0 & -1 & 0 \\
1 & 0 & -1 & 0 & 0 & 0 \\
0 & 0 & 0 & 0 & 0 & -1
\end{array}\right)\left(\begin{array}{c}
\sigma_{\eta, 1}^{2} \\
\sigma_{\eta, 2}^{2} \\
\sigma_{\omega, 1}^{2} \\
\sigma_{\omega, 2}^{2} \\
\sigma_{\varepsilon, 1}^{2} \\
\sigma_{\varepsilon, 2}^{2}
\end{array}\right)=\left(\begin{array}{c}
\Gamma_{0}(1,1) \\
\Gamma_{0}(1,2) \\
\Gamma_{0}(2,2) \\
\Gamma_{1}(1,1) \\
\Gamma_{1}(1,2) \\
\Gamma_{1}(2,2)
\end{array}\right),
$$

where $\Gamma_{j}(i, k)$ indicates the element on row $i$ and column $k$ of matrix $\Gamma_{j}$. The system of equations (29) clearly has multiple solutions as the the first matrix only has rank 5 . The null space of this matrix is spanned by the vector $\left(\begin{array}{llllll}1 & -1 & 1 & -1 & 0 & 0\end{array}\right)^{\prime}$. Hence, we have to impose a restriction on the first four parameters to obtain identification, for example

$$
\sigma_{\eta, 1}^{2}-\sigma_{\eta, 2}^{2}+\sigma_{\omega, 1}^{2}-\sigma_{\omega, 2}^{2}=0
$$

Note that the restriction is not unique, we can also impose

$$
\sigma_{\eta, 1}^{2}=\sigma_{\eta, 2}^{2} \quad \text { or } \quad \sigma_{\omega, 1}^{2}=\sigma_{\omega, 2}^{2}
$$

The identification problem for $S=2$ also applies to extended versions of the model. The identification of the model with a local linear trend is discussed in Appendix A. In the periodic local linear trend model, we have $4 S$ parameters: trend, slope, seasonal and irregular variances, and $2 S^{2}$ linear equations, so for $S=2$ we get 8 equations for 8 parameters, but we get a similar identification problem as in model (6) as the rank condition is not satisfied.

The reduced rank problem as described above for $S=2$ does not occur when $S>2$. For the periodic BSM in equation (6) we have $S(S+1)$ linear equations to estimate $3 S$ parameters. It is clear that for $S \geq 3$ we have more nonzero moment equations than parameters, so the order condition for identification is satisfied. Moreover, only $3 S$ unique equations exist in the system. 
Therefore all parameters are exactly identified. This result extends to the periodic local linear trend model. We conclude that the parameters are exactly identified in these two standard periodic unobserved component models for all $S>2$.

The parameters of the periodic UC models are estimated by Gaussian ML. The exact likelihood of the model is efficiently obtained by the Kalman Filter based on the prediction error decomposition of the Gaussian model. We use diffuse initialisation for nonstationary states to obtain the exact likelihood. Further, we employ the Broyden-Fletcher-Goldfarb-Shanno (BFGS) numerical optimisation algorithm, c.f. Fletcher (1987), to maximise with respect to the unknown parameters. The number of parameters in periodic models is large for a high value of $S$. However, this is not a problem in practice if the time series is sufficiently long, as we illustrate below in $\S 4.3$.

\subsection{Periodic stochastic cycle model}

Macroeconomic time series often require a cyclical component in their specification. In this section we introduce a novel periodic version of the stochastic cycle component as part of the UC time series models. We extend equation (5) by having $\rho$ and $\sigma_{\kappa}^{2}$ periodic. To save space we only present the equations for a model with two periodic components, cycle and irregular, and with $S=2$. We define for $t=1, S+1,2 S+1, \ldots,\left(n^{*}-1\right) S+1$ :

$$
\begin{array}{lll}
y_{t}=\psi_{t}+\varepsilon_{t}, & & \varepsilon_{t} \sim \operatorname{NID}\left(0, \sigma_{\varepsilon, 1}^{2}\right) \\
y_{t+1}=\psi_{t+1}+\varepsilon_{t+1}, & & \varepsilon_{t+1} \sim \operatorname{NID}\left(0, \sigma_{\varepsilon, 2}^{2}\right),
\end{array}
$$

where

$$
\begin{aligned}
& \left(\begin{array}{c}
\psi_{t+1} \\
\psi_{t+1}^{+}
\end{array}\right)=\rho_{1}\left(\begin{array}{cc}
\cos \lambda & \sin \lambda \\
-\sin \lambda & \cos \lambda
\end{array}\right)\left(\begin{array}{c}
\psi_{t} \\
\psi_{t}^{+}
\end{array}\right)+\left(\begin{array}{c}
\kappa_{t} \\
\kappa_{t}^{+}
\end{array}\right), \quad \kappa_{t}, \kappa_{t}^{+} \sim \operatorname{NID}\left(0, \sigma_{\kappa, 1}^{2}\right) \\
& \left(\begin{array}{c}
\psi_{t+2} \\
\psi_{t+2}^{+}
\end{array}\right)=\rho_{2}\left(\begin{array}{cc}
\cos \lambda & \sin \lambda \\
-\sin \lambda & \cos \lambda
\end{array}\right)\left(\begin{array}{c}
\psi_{t+1} \\
\psi_{t+1}^{+}
\end{array}\right)+\left(\begin{array}{c}
\kappa_{t+1} \\
\kappa_{t+1}^{+}
\end{array}\right), \quad \kappa_{t+1}, \kappa_{t+1}^{+} \sim \operatorname{NID}\left(0, \sigma_{\kappa, 2}^{2}\right),
\end{aligned}
$$

with mutually uncorrelated white noise disturbances $\left(\kappa_{t}, \kappa_{t+1}\right)$ and $\left(\kappa_{t}^{+}, \kappa_{t+1}^{+}\right)$. A restriction on the damping terms $0<\prod_{s=1}^{S} \rho_{s}<1$ ensures that the stochastic process $\psi_{t}$ is stationary. The overall frequency of the stochastic cycle is fixed at $0<\lambda<\pi$ for all seasons $s$. This implies that the average period of the cycle is $2 \pi / \lambda$ in terms of $t$, i.e. semesters for $S=2$, and equal to $2 \pi /(\lambda S)$ years.

The dynamic properties of the stationary periodic cycle process are given by expressions for the variances and covariances of $\psi_{t}$ and $\psi_{t+1}$. These are derived from their vector autoregressive representation of order $1, \operatorname{VAR}(1)$. This yearly $\operatorname{VAR}(1)$ is obtained by substituting the expression 
for $\left(\psi_{t+1}^{+}, \psi_{t+1}^{+}\right)^{\prime}$ in the second equation of (32). We get:

$$
\begin{aligned}
& \Psi_{t^{*}+1}=\Phi \Psi_{t^{*}}+\kappa_{t^{*}} \\
&\left(\begin{array}{c}
\psi_{t+2} \\
\psi_{t+2}^{+} \\
\kappa_{t+2} \\
\kappa_{t+2}^{+}
\end{array}\right)=\left(\begin{array}{cccc}
\rho_{1} \rho_{2} \cos 2 \lambda & \rho_{1} \rho_{2} \sin 2 \lambda & \rho_{2} \cos \lambda & \rho_{2} \sin \lambda \\
-\rho_{1} \rho_{2} \sin 2 \lambda & \rho_{1} \rho_{2} \cos 2 \lambda & -\rho_{2} \sin \lambda & \rho_{2} \cos \lambda \\
0 & 0 & 0 & 0 \\
0 & 0 & 0 & 0
\end{array}\right)\left(\begin{array}{c}
\psi_{t} \\
\psi_{t}^{+} \\
\kappa_{t} \\
\kappa_{t}^{+}
\end{array}\right)+\left(\begin{array}{c}
\kappa_{t+1} \\
\kappa_{t+1}^{+} \\
\kappa_{t+2} \\
\kappa_{t+2}^{+}
\end{array}\right),
\end{aligned}
$$

for $t^{*}=1,2, \ldots, n^{*}$ and for $t=1, S+1,2 S+1, \ldots,\left(n^{*}-1\right) S+1$. First we derive the variance covariance matrix $\Lambda_{0}$ of $\Psi_{t^{*}}$ :

$$
\begin{aligned}
\mathbb{E}\left[\Psi_{t^{*}+1} \Psi_{t^{*}+1}^{\prime}\right] & =\Phi \mathbb{E}\left[\Psi_{t^{*}} \Psi_{t^{*}}^{\prime}\right] \Phi^{\prime}+\Sigma_{\kappa} \Leftrightarrow \\
\Lambda_{0} & =\Phi \Lambda_{0} \Phi^{\prime}+\Sigma_{\kappa},
\end{aligned}
$$

where

$$
\Sigma_{\kappa}=\operatorname{diag}\left(\begin{array}{cccc}
\sigma_{\kappa, 2}^{2} & \sigma_{\kappa, 2}^{2} & \sigma_{\kappa, 1}^{2} & \sigma_{\kappa, 1}^{2}
\end{array}\right)
$$

and

$$
\Lambda_{0}=\left(\begin{array}{cccc}
\sigma_{\psi, 1}^{2} & \mathbb{E}\left(\psi_{t} \psi_{t}^{+}\right) & 0 & 0 \\
\mathbb{E}\left(\psi_{t} \psi_{t}^{+}\right) & \sigma_{\psi, 1}^{2} & 0 & 0 \\
0 & 0 & \sigma_{\kappa, 1}^{2} & 0 \\
0 & 0 & 0 & \sigma_{\kappa, 1}^{2}
\end{array}\right)
$$

with

$$
\begin{aligned}
\mathbb{E}\left(\psi_{t} \psi_{t}^{+}\right) & =0, & \sigma_{\psi, 1}^{2} & =\frac{\sigma_{\kappa, 2}^{2}+\rho_{2}^{2} \sigma_{\kappa, 1}^{2}}{1-\rho_{1}^{2} \rho_{2}^{2}}, \\
\mathbb{E}\left(\psi_{t+1} \psi_{t+1}^{+}\right) & =0, & \sigma_{\psi, 2}^{2} & =\frac{\sigma_{\kappa, 1}^{2}+\rho_{1}^{2} \sigma_{\kappa, 2}^{2}}{1-\rho_{1}^{2} \rho_{2}^{2}} .
\end{aligned}
$$

Subsequently, the periodic autocovariance function (ACF) of $y_{t}$ for $S=2, t=1, S+1,2 S+1, \ldots$ and $t^{*}=1,2, \ldots$ is expressed in terms of $\sigma_{\psi, s}^{2}, \sigma_{\varepsilon, s}^{2}, \rho_{s}$ and $\lambda$ :

$$
\begin{aligned}
& \Gamma_{0}=\mathbb{E}\left[\left(\begin{array}{c}
y_{t} \\
y_{t+1}
\end{array}\right)\left(\begin{array}{c}
y_{t} \\
y_{t+1}
\end{array}\right)^{\prime}\right]=\mathbb{E}\left[\left(\begin{array}{c}
y_{1, t^{*}} \\
y_{2, t^{*}}
\end{array}\right)\left(\begin{array}{l}
y_{1, t^{*}} \\
y_{2, t^{*}}
\end{array}\right)^{\prime}\right]=\left(\begin{array}{cc}
\sigma_{\psi, 1}^{2}+\sigma_{\varepsilon, 1}^{2} & \rho_{1} \cos (\lambda) \sigma_{\psi, 1}^{2} \\
\rho_{1} \cos (\lambda) \sigma_{\psi, 1}^{2} & \sigma_{\psi, 2}^{2}+\sigma_{\varepsilon, 2}^{2}
\end{array}\right) \\
& \Gamma_{1}=\mathbb{E}\left[\left(\begin{array}{c}
y_{t} \\
y_{t+1}
\end{array}\right)\left(\begin{array}{c}
y_{t-2} \\
y_{t-1}
\end{array}\right)^{\prime}\right]=\mathbb{E}\left[\left(\begin{array}{c}
y_{1, t^{*}} \\
y_{2, t^{*}}
\end{array}\right)\left(\begin{array}{c}
y_{1, t^{*}-1} \\
y_{2, t^{*}-1}
\end{array}\right)^{\prime}\right]=\left(\begin{array}{cc}
\rho_{1} \rho_{2} \cos (2 \lambda) \sigma_{\psi, 1}^{2} & \rho_{2} \cos (\lambda) \sigma_{\psi, 2}^{2} \\
\rho_{1}^{2} \rho_{2} \cos (3 \lambda) \sigma_{\psi, 1}^{2} & \rho_{1} \rho_{2} \cos (2 \lambda) \sigma_{\psi, 2}^{2}
\end{array}\right) \\
& \Gamma_{j}=\mathbb{E}\left[\left(\begin{array}{c}
y_{t} \\
y_{t+1}
\end{array}\right)\left(\begin{array}{c}
y_{t-2 j} \\
y_{t+1-2 j}
\end{array}\right)^{\prime}\right]=\mathbb{E}\left[\left(\begin{array}{l}
y_{1, t^{*}} \\
y_{2, t^{*}}
\end{array}\right)\left(\begin{array}{l}
y_{1, t^{*}-j} \\
y_{2, t^{*}-j}
\end{array}\right)^{\prime}\right] \\
& =\left(\begin{array}{cc}
\rho_{1}^{j} \rho_{2}^{j} \cos (2 j \lambda) \sigma_{\psi, 1}^{2} & \rho_{1}^{j-1} \rho_{2}^{j} \cos ((2 j-1) \lambda) \sigma_{\psi, 2}^{2} \\
\rho_{1}^{j+1} \rho_{2}^{j} \cos ((2 j+1) \lambda) \sigma_{\psi, 1}^{2} & \rho_{1}^{j} \rho_{2}^{j} \cos (2 j \lambda) \sigma_{\psi, 2}^{2}
\end{array}\right) \quad j=1,2, \ldots
\end{aligned}
$$


The autocovariance function of $y_{t}$ has a nonlinear structure in terms of $\sigma_{\psi, s}, \rho_{s}$ and $\lambda$ with $s=1,2$. Since $\sigma_{\psi, s}$ also depends on $\sigma_{\kappa, s}$ and $\rho_{s}$, the structure of the ACF becomes intricate. As a result, identification can not be analysed analytically and we therefore carry out some Monte Carlo experiments for Maximum Likelihood estimation to investigate whether the parameters can be estimated from simulated data. Model (32) is already in state space form, which makes it very suitable for exact ML estimation. However, the construction of the exact likelihood function of models with a periodic cyclical component involves one non-standard step. The expression for $\sigma_{\psi, 1}^{2}$ is needed for the exact initialisation, since $\left(\psi_{1}, \psi_{1}^{+}\right)$is included in the state vector corresponding to the first observation. The other terms in the likelihood for $t=2, \ldots$ follow in a standard way from the periodic Kalman Filter equations.
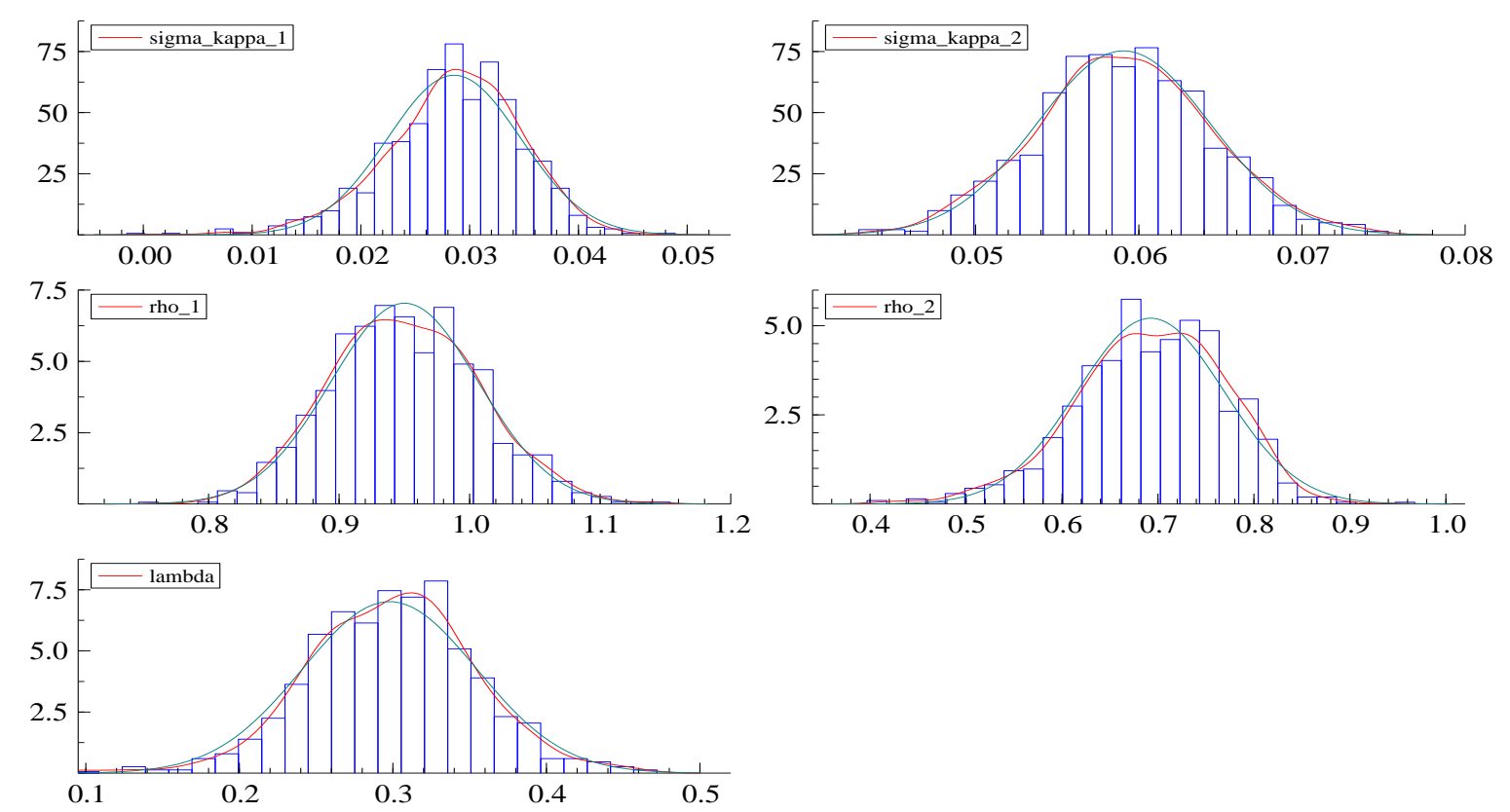

Figure 1: Simulated densities and histograms of the exact ML parameter estimates in periodic cycle model (32) with true values: $\sigma_{\kappa, 1}=0.03, \sigma_{\kappa, 2}=0.06, \rho_{1}=0.95, \rho_{2}=0.70$ and $\lambda=0.3$. Based a on Monte Carlo experiment of 1000 simulations with 100 observations.

The Monte Carlo experiments are implemented for different values for the parameters $\sigma_{\kappa, 1}, \sigma_{\kappa, 2}$, $\rho_{1}, \rho_{2}$ and $\lambda$. We look at the finite sample distribution of the exact ML estimators of these parameters and run this experiments 1000 times with 100 observations. All computations and graphs in this paper are made using recent versions of SsfPack, see Koopman, Shephard \& Doornik (1999) and Ox, see Doornik (1999). Figure 1 reports the simulated densities for one representative set of parameter values, based on empirical estimates for half-yearly postwar data of $\log$ US unemployment. We see that the simulated means lie around the true values and the 
simulated densities are approximately normal. This confirms that a periodic cycle in a periodic model does not lead to additional identification problems for parameter estimation, not even for $S=2$. Of course in larger models, we have to exclude extreme cases where the parameters $\rho_{s}$ are nearly one and $\lambda$ is very close to $\pi$, to avoid confusion with the seasonal component.

The extension of the periodic cycle model to a general $S=2,3, \ldots$ is straightforward. The $\Phi$ matrix in the multivariate $\operatorname{VAR}(1)$ form (33) becomes

$$
\left(\begin{array}{cccc}
\prod_{i=1}^{S} \rho_{i} C(S \lambda) & \prod_{i=2}^{S} \rho_{i} C((S-1) \lambda) & \ldots & \rho_{S} C(\lambda) \\
& \boldsymbol{O}_{2(S-1) \times 2 S} & &
\end{array}\right)
$$

where

$$
C(i \lambda)=\left(\begin{array}{cc}
\cos i \lambda & \sin i \lambda \\
-\sin i \lambda & \cos i \lambda
\end{array}\right)
$$

for $i=1, \ldots, S$ and the general solution for $\sigma_{\psi, 1}^{2}$ in (34) becomes

$$
\sigma_{\psi, 1}^{2}=\frac{\sum_{i=1}^{S-1}\left\{\sigma_{\kappa, i}^{2}\left[\prod_{j=i+1}^{S} \rho_{j}^{2}\right]\right\}+\sigma_{\kappa, S}^{2}}{1-\prod_{i=1}^{S} \rho_{i}^{2}} .
$$

The construction of the state space form for more extensive models with a periodic stochastic cycle is not problematic. Adding a cyclical component to the $T^{*}, Z^{*}$ and $H^{*}$ matrices in $\S 3.2$ is easily done, as we assume independence between the unobserved components, $\mu_{t}, \gamma_{t}$ and $\psi_{t}$.

\subsection{Model summary}

In sum, we have analysed the following periodic unobserved components model for decomposing and forecasting univariate periodic time series:

$$
y_{t}=\mu_{t}+\gamma_{t}+\psi_{t}+\varepsilon_{t}, \quad t=1, \ldots, n
$$

where $\mu_{t}$ and $\gamma_{t}$ are defined in (3) and (4), with disturbance variances $\sigma_{\eta, s}^{2}, \sigma_{\zeta, s}^{2}$, and $\sigma_{\omega, s}^{2}$, where $\psi_{t}$ is defined in (32), where $\varepsilon_{t}$ has variances $\sigma_{\varepsilon, s}^{2}$, where all disturbances are independent, $s=1, \ldots, S$ and $n=n^{*} S$. Periodic trigonometric seasonality instead of dummy variable seasonality can be specified in this framework by a set of periodic stochastic cycles with fixed seasonal frequencies $\lambda_{j}=2 \pi j / S, j=1, \ldots, S / 2$, where the corresponding damping factors $\rho_{j, s}$ might be fixed at one to allow nonstationary seasonality. Parameter estimates, time series decompositions, diagnostics and forecasts are based on the Gaussian state space form of the model that we discussed in $\S 3.2$. We use exact maximum likelihood estimation with diffuse 
initialisation of the nonstationary state variables. Conditional on the ML estimates of the hyperparameters, $\hat{\sigma}_{\eta, s}, \hat{\sigma}_{\zeta, s}, \hat{\sigma}_{\omega, s} \hat{\rho}_{s}, \hat{\sigma}_{\kappa, s}$ and $\hat{\lambda}$, we compute the decomposition into trend, seasonal, cycle and irregular components as

$$
\hat{\mu}_{t}=\mathbb{E}\left(\mu_{t} \mid y_{1}, \ldots, y_{n}\right), \quad \hat{\gamma}_{t}=\mathbb{E}\left(\gamma_{t} \mid y_{1}, \ldots, y_{n}\right), \quad \hat{\psi}_{t}=\mathbb{E}\left(\psi_{t} \mid y_{1}, \ldots, y_{n}\right),
$$

and

$$
\hat{\varepsilon}_{t}=\mathbb{E}\left(\varepsilon_{t} \mid y_{1}, \ldots, y_{n}\right)
$$

respectively. The seasonally adjusted series is defined as $y_{t}-\hat{\gamma}_{t}$. The diagnostics are based on the one-step-ahead forecast errors

$$
v_{t}=y_{t}-\mathbb{E}\left(y_{t} \mid y_{1}, \ldots, y_{t-1}\right)
$$

that are scaled by their standard errors

$$
\text { s.e. }\left(v_{t}\right)=\left[\mathbb{E}\left(v_{t}^{2}\right) \mid y_{1}, \ldots, y_{t-1}\right]^{1 / 2} \text {. }
$$

The optimal linear out-of-sample forecasts are computed as

$$
\hat{y}_{n+i}=\mathbb{E}\left(y_{n+i} \mid y_{1}, \ldots, y_{n}\right), \quad i=1,2, \ldots
$$

\section{Application to US unemployment data}

\subsection{Data analysis}

In this section we analyse a monthly time series of US unemployment data using both periodic seasonal autoregressive integrated moving average (SARIMA) and periodic unobserved components time series models. The data consists of seasonally unadjusted monthly US unemployment levels (in thousands of persons) for age 16 years or over. The estimation sample, 1948.1-2005.12, has 696 observations. We use 2006.1-2006.8 to evaluate forecasts. This series is published by the US Bureau of Labor Statistics (BLS) and is obtained via www.economagic.com.

Figure 2 presents time series plots of log monthly unemployment, $y_{t}$. The top panel shows the data month by month, while the bottom panel presents the data in multivariate form, year by year for each month. The monthly unemployment series is slowly trending upwards and contains a pronounced cyclical pattern. Although the plots of the series in the bottom panel do not contain seasonal movements, this does not mean that the seasonal dependence has disappeared. The yearly series are smoother than the monthly series and have clearly common dynamics. 

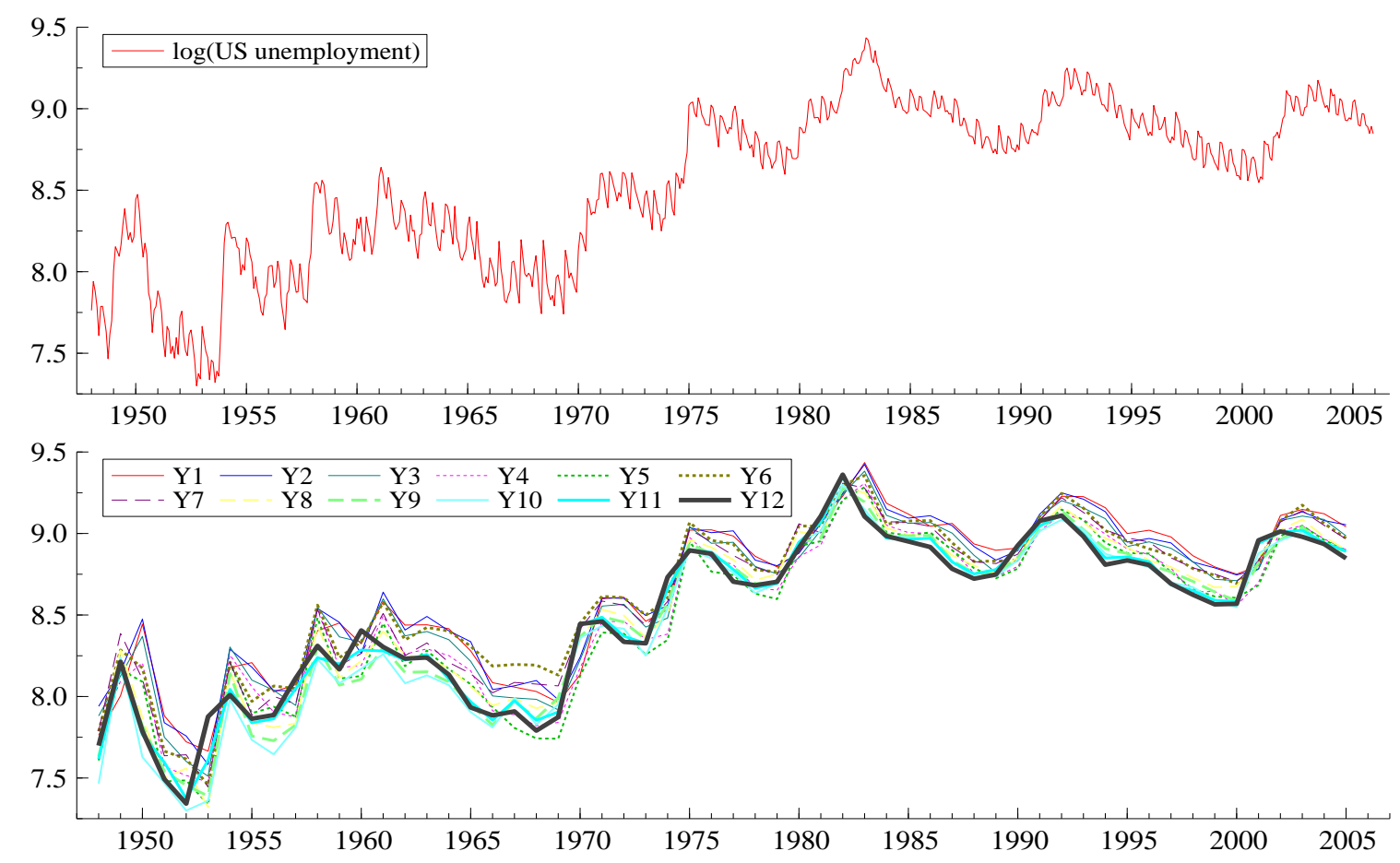

Figure 2: US unemployment (in logs, seasonally unadjusted), 1948.1 - 2005.12. Top: Monthly time series, Bottom: Yearly time series for each month of the year, $s=1, \ldots, 12$.

Figure 3 shows a selection of the periodic sample autocorrelations of yearly changes in US unemployment growth rates, $\Delta \Delta_{12} y_{t}$. Their precise definition is given in Appendix C. These sample autocorrelation coefficients are clearly periodic. They differ significantly from month to month. For example, for March (top left panel), there is a pronounced short cyclical movement of approximately 24 months, while we see a much longer cyclical movement for June (top right panel). There is also a significant difference between March and June for the seasonal autocorrelation at lag 12. The periodicity in the autocorrelation structure is the main motivation for periodic modelling of log US unemployment. The autocorrelations indicate the need for a periodic cyclical component and a periodic seasonal component in time series models.

\subsection{Periodic SARIMA model for US unemployment}

For a closer statistical examination of the dynamic properties of the series, we first model the monthly US unemployment dataset using a reduced form periodic seasonal autoregressive integrated moving average (SARIMA) model. Periodic SARIMA models provide good benchmarks for periodic unobserved component (UC) time series models. They provide an alternative framework for tests for periodicity and for forecasting ability, although they do not allow for time series 

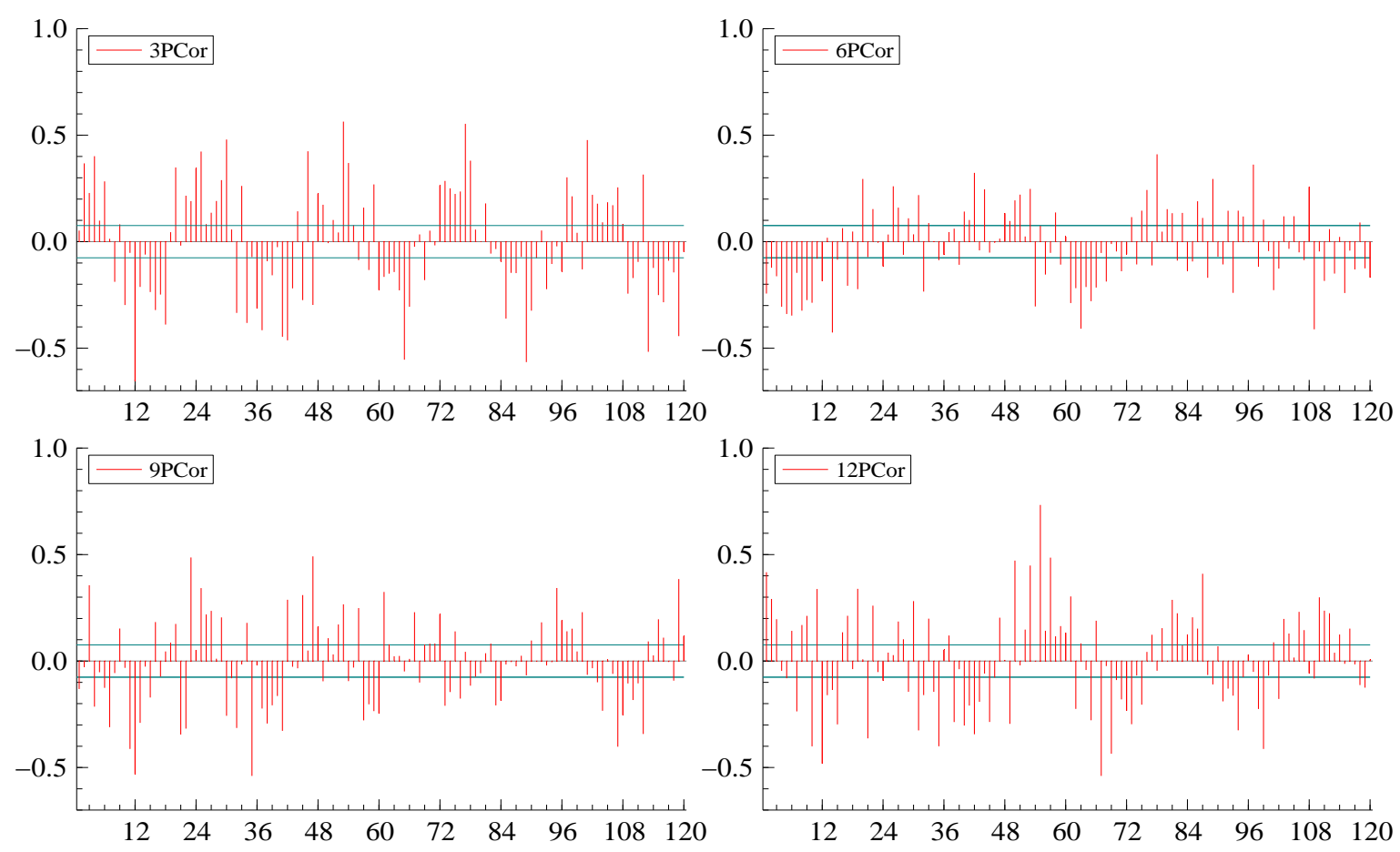

Figure 3: Periodic autocorrelations of $\Delta_{12} y_{t}$ : monthly changes in the yearly growth rates of US unemployment, 1949.1-2005.12. Autocorrelations for lags of 1 to 120 months, for $s=3,6,9,12$. decomposition yet. Moreover, as we have argued above, periodic UC models can be interpreted as restricted periodic SARIMA models.

Empirically, we obtain our best SARIMA results for the seasonally differenced series, $y_{t}^{\dagger}=\Delta_{12} y_{t}$. We define $\beta_{s}$ as the yearly growth rate of $y_{t}$ in season $s$, so we allow for periodic slopes in the long term trend. In our specification $y_{t}^{\dagger}$ follows a periodic $\operatorname{SARIMA}(2,0,1)(0,0,1)_{12}$ process, that is, for $t=13, \ldots, n$ :

$$
y_{t}^{\dagger}=\beta_{s}+\phi_{1, s}\left(y_{t-1}^{\dagger}-\beta_{s}\right)+\phi_{2, s}\left(y_{t-2}^{\dagger}-\beta_{s}\right)+\varepsilon_{t}+\theta_{1, s} \varepsilon_{t-1}+\Theta_{1, s} \varepsilon_{t-12}+\theta_{1, s} \Theta_{1, s} \varepsilon_{t-13}
$$

where $\varepsilon_{t} \sim N\left(0, \sigma_{\varepsilon, s}^{2}\right)$ and where $\beta_{s}, \phi_{1, s}, \phi_{2, s}, \theta_{1, s}, \Theta_{1, s}$ and $\sigma_{\varepsilon, s}^{2}$ apply when $y_{t}^{\dagger}$ falls in period $s$, and $n=n^{*} S$. In our application we simplify further by dropping the term associated with $\varepsilon_{t-13}$. This has not led to a significant deterioration of the model fit.

In order to facilitate comparison with the periodic UC model we cast the periodic SARIMA model (39) directly in a state space form for $y_{t}$. This requires the modification and extension of existing SARIMA state space forms for differenced series, as employed by e.g. Jimenez et al. (1989). The sample for $y_{t}$ starts at $t=1$ and nonstationary elements of the state vector are initialised with a diffuse distribution in order to obtain an exact likelihood which can be compared with the likelihoods for the periodic UC model. The details are presented in Appendix D. 
In sum, $y_{t}$ is specified as a restricted periodic $\operatorname{SARIMA}(2,0,1)(0,1,1)_{12}$ model with 72 unknown parameters, $\beta_{s}, \phi_{1, s}, \phi_{2, s}, \theta_{1, s}, \Theta_{1, s}$ and $\sigma_{\varepsilon, s}$ for $s=1,2, \ldots, 12$. Given the state space form for $y_{t}$, maximum likelihood estimation of $\phi_{1, s}, \phi_{2, s}, \theta_{1, s}, \Theta_{1, s}$ and $\sigma_{\varepsilon, s}$ and the computation of forecasts and diagnostics is based on the same procedures as discussed in $\S 3.5$. The periodic growth rates, $\beta_{s}$, are included in the state vector. In this way, they are effectively concentrated out of the likelihood function. Although the remaining number of 60 parameters is large, it should be emphasised that we have more than 50 years of monthly data. Empirically, it turns out to be quite feasible to estimate this model.
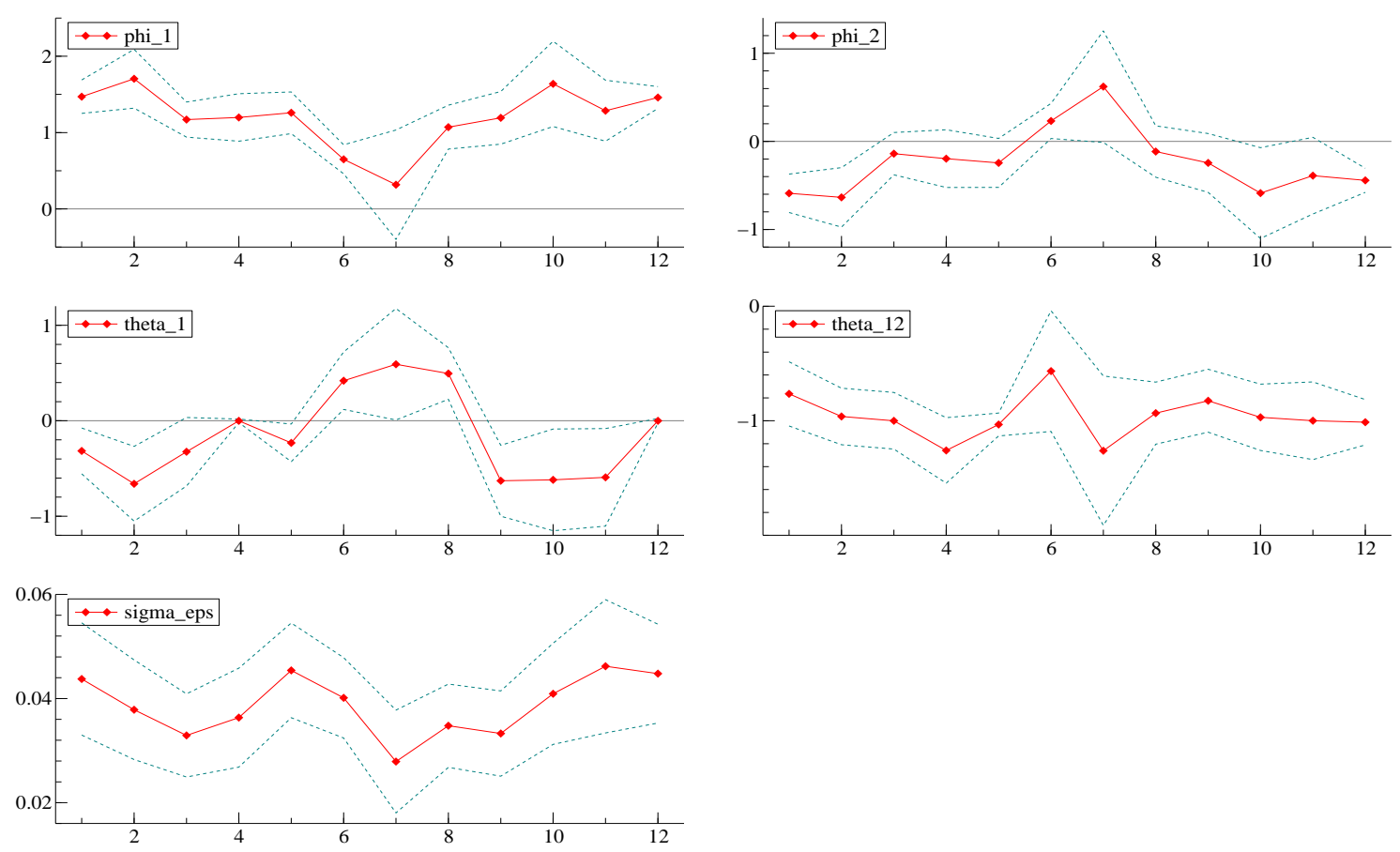

Figure 4: Estimated parameters for periodic SARIMA model (39) for log US unemployment with $s=1, \ldots, 12$. Top: $\hat{\phi}_{1, s}, \hat{\phi}_{2, s}$. Middle: $\hat{\theta}_{1, s}, \hat{\Theta}_{1, s}$. Bottom: $\hat{\sigma}_{\varepsilon, s}$. All estimated parameters are plotted with \pm 2 standard errors.

Figure 4 gives a graphical presentation of the parameter estimates of the periodic SARIMA model for US unemployment with \pm 2 standard errors. We see significant fluctuations in parameter estimates across the different months of the year which suggests that a non-periodic SARIMA specification is implausibly restrictive.

The possible presence of a cycle in the model can be derived from the eigenvalues of the AR-part of the periodic SARIMA model, analogous to the eigenvalues of $\Phi$ in (33). In our case, we obtain the two real eigenvalues 0.00 and 0.63 . This implies that the model does not capture cyclical dynamics. 
Figure 5 shows graphical diagnostics for the periodic SARIMA model and for our periodic UC model that we discuss in the next subsection. The top row of Figure 5 shows the standardised one-step ahead prediction errors of the periodic SARIMA model, together with the sample autocorrelation function, a histogram and a density estimate. Most aspects of these diagnostics appear to be satisfactory, but there is one notable exception: the forecast errors are clearly more volatile in the first half of the series compared to the second half. This is an important point that we address in $\S 4.4$.
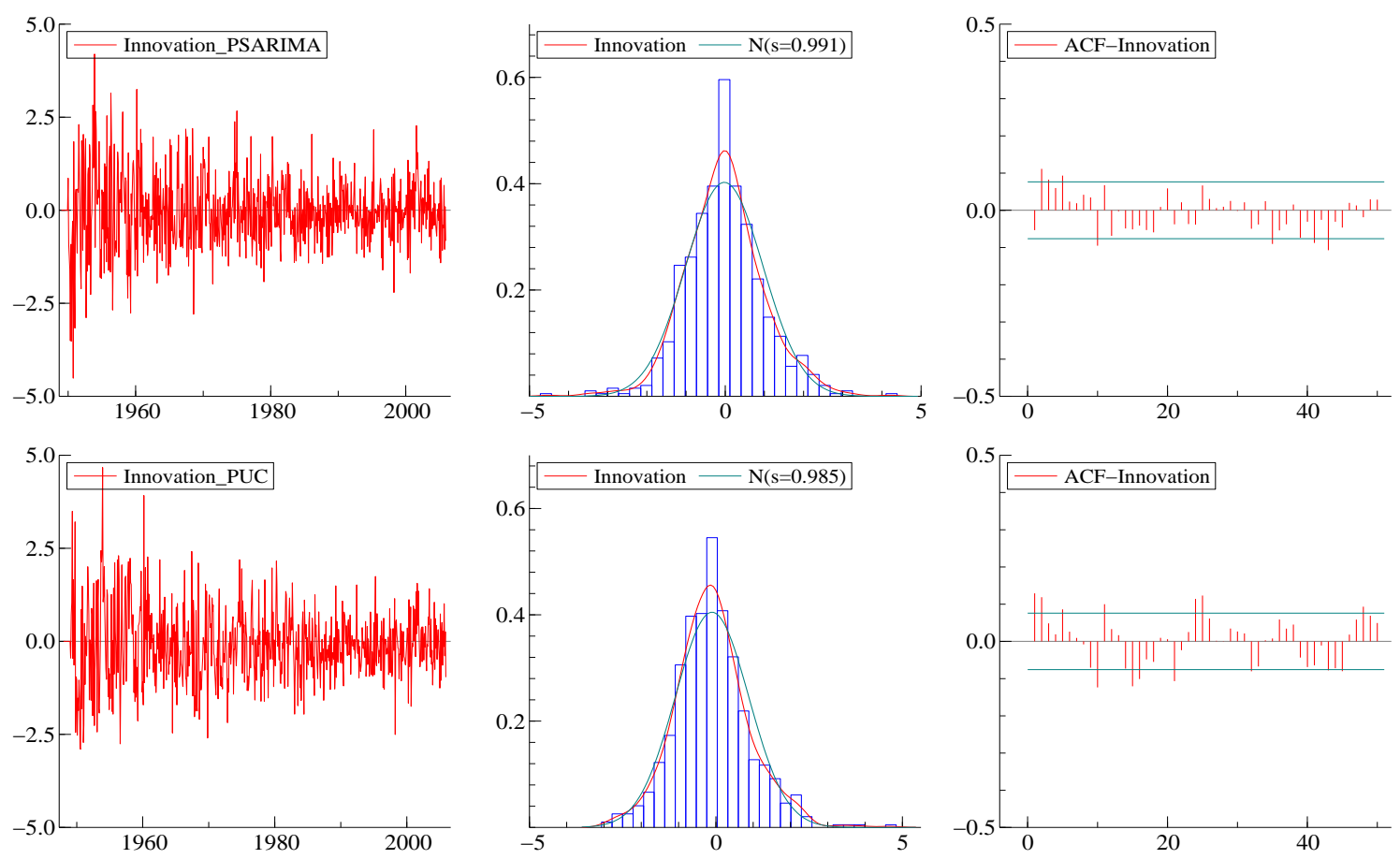

Figure 5: Time series plot of scaled one-step-ahead prediction errors, their density and sample ACF for the periodic SARIMA model (top panel) and for the periodic UC model (bottom panel). Note the heteroskedasticity in both sets of residuals.

\subsection{Periodic unobserved component model for US unemployment}

We have shown thau US unemployment is subject to periodic dynamics and therefore we continue our analysis by considering a periodic UC model, consisting of trend, season and cycle components as given in (38). The disturbance variances of the trend, season, cycle and irregular components are all periodic. The model is cast into a state space form containing $6 S+1$ known parameters, $\sigma_{\eta, s}, \sigma_{\zeta, s}, \sigma_{\omega, s}, \sigma_{\kappa, s}, \sigma_{\varepsilon, s}, \rho_{s}$ and $\lambda$ for $s=1, \ldots, S$. The parameters are estimated by numerically maximising the exact loglikelihood. In our case with $S=12$, we have to estimate 73 parameters. The parameters $\sigma_{\eta, s}$ and $\sigma_{\varepsilon, s}$ are estimated at zero for all seasons. For $\sigma_{\zeta, s}$ 
we obtain one nonzero estimate at $s=9$. We present estimated components and associated parameters in Figures 6, 7, and 8.

Figure 6 presents the estimated trend component and the seasonally adjusted series. The top panel shows the trend, $\hat{\mu}_{t}$, together with the unadjusted observations, $y_{t}$. In the bottom panel we show the trend and the seasonally adjusted data, $y_{t}-\hat{\gamma}_{t}$. The trend is very smooth, it increases steadily until early 1980 and flattens out afterwards. The difference between the two series in the bottom graph, $y_{t}-\hat{\gamma}_{t}-\hat{\mu}_{t}$ can be interpreted as the estimated cycle, $\hat{\psi}_{t}$, as the irregular component, $\hat{\varepsilon}_{t}$, is estimated as zero.

Figure 7 shows the estimated slope, $\hat{\beta}_{t}$, and the cyclical component, $\hat{\psi}_{t}$. The corresponding parameters are shown underneath. The slope has a somewhat surprising shape, jumping in discrete steps. This occurs because the slope is a random walk with zero innovations for all months except October $(s=9)$. Further, the slope remains stepwise periodic throughout the sample but it becomes nearly constant after 1982, which results in a smoother trend. The cyclical component shows large swings throughout the whole sample.
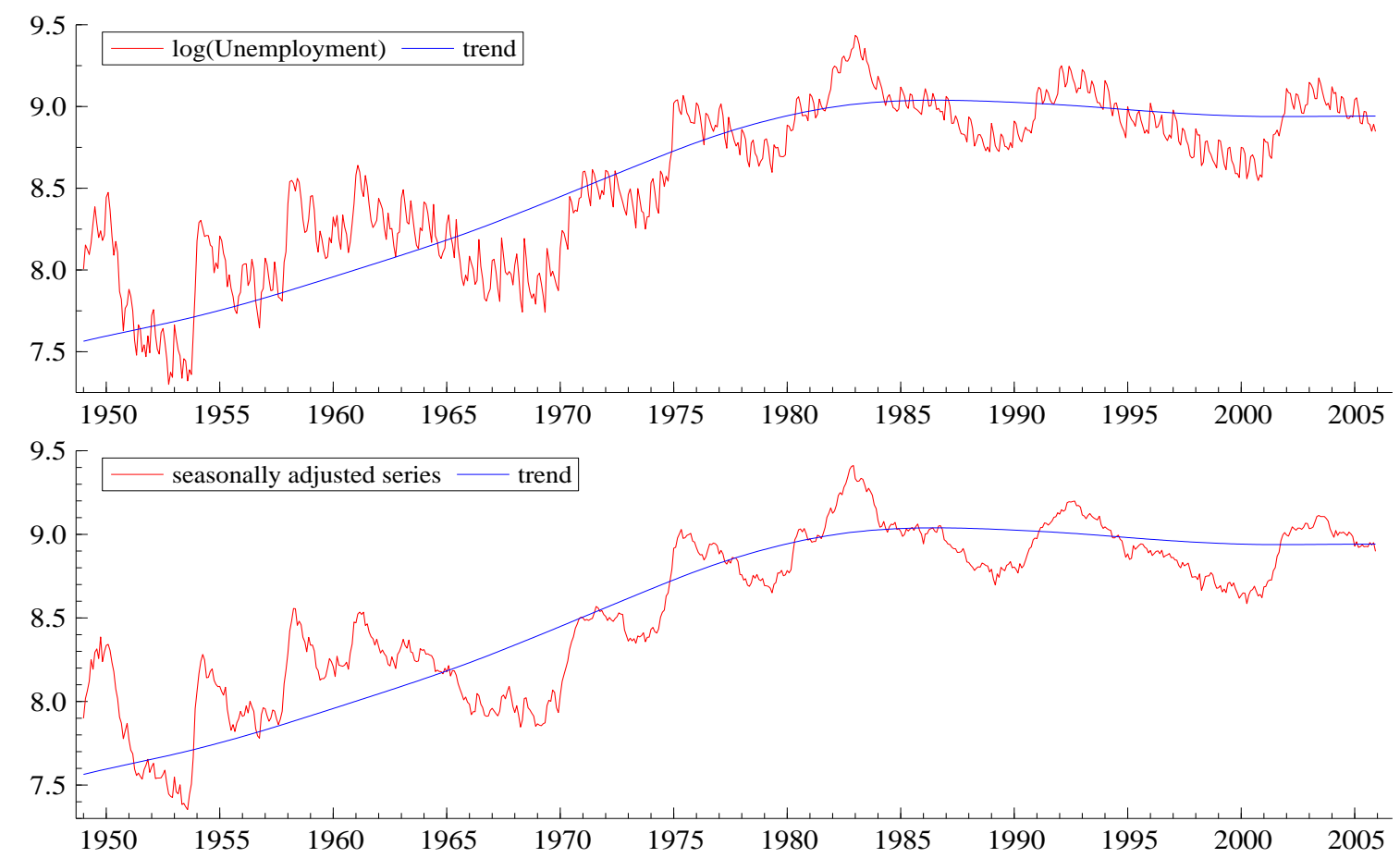

Figure 6: Top panel: log of US unemployment with the estimated trend from the periodic UC model. Bottom panel: the model based seasonally adjusted series together with the estimated trend.

The bottom panel of Figure 7 shows the estimated parameters for the trend and cycle, where we present $\sigma_{\zeta, s}, \sigma_{\kappa, s}$ and $\rho_{s}$ for $s=1, \ldots, 12$. The estimated slope parameters, $\sigma_{\zeta, s}$, show a 
particular form of periodicity, they are all zero except for $\sigma_{\zeta, 9}$. This might be interpreted as a structural change in the unemployment trend due to newcomers on the job market in October. Note that the nonzero $\sigma_{\zeta, 9}$ in the bottom left graph of Figure 7 corresponds to the slope in month 10, hence October, as $\beta_{t+1}=\beta_{t}+\zeta_{t}$ with $\zeta_{t} \sim \operatorname{NID}\left(0, \sigma_{\zeta, s}^{2}\right)$. The damping factors of the cycle, $\rho_{s}$, look periodic as they have different values for each month and vary between 0.8 and 1.1. Despite the fact that some $\rho$ 's are estimated to be larger than one, the cyclical component is still stationary as the product of all the $\rho$ 's is less than one. The lowest $\rho$ is found in June $\left(\rho_{5}\right)$ and the highest in December $\left(\rho_{11}\right)$. Note that $\rho_{1}$ is linked with the transition equation for February and $\rho_{12}$ appears in the equation for January. The shocks in the cyclical component have periodic standard errors that vary between 0.03 and 0.07 throughout the year. To conclude our discussion of the trend and cycle estimates we report that $\hat{\lambda}=0.049$ with a standard error of 0.013 . This corresponds to an estimate of the average cycle length of 10.7 with a standard error of 2.8 years.
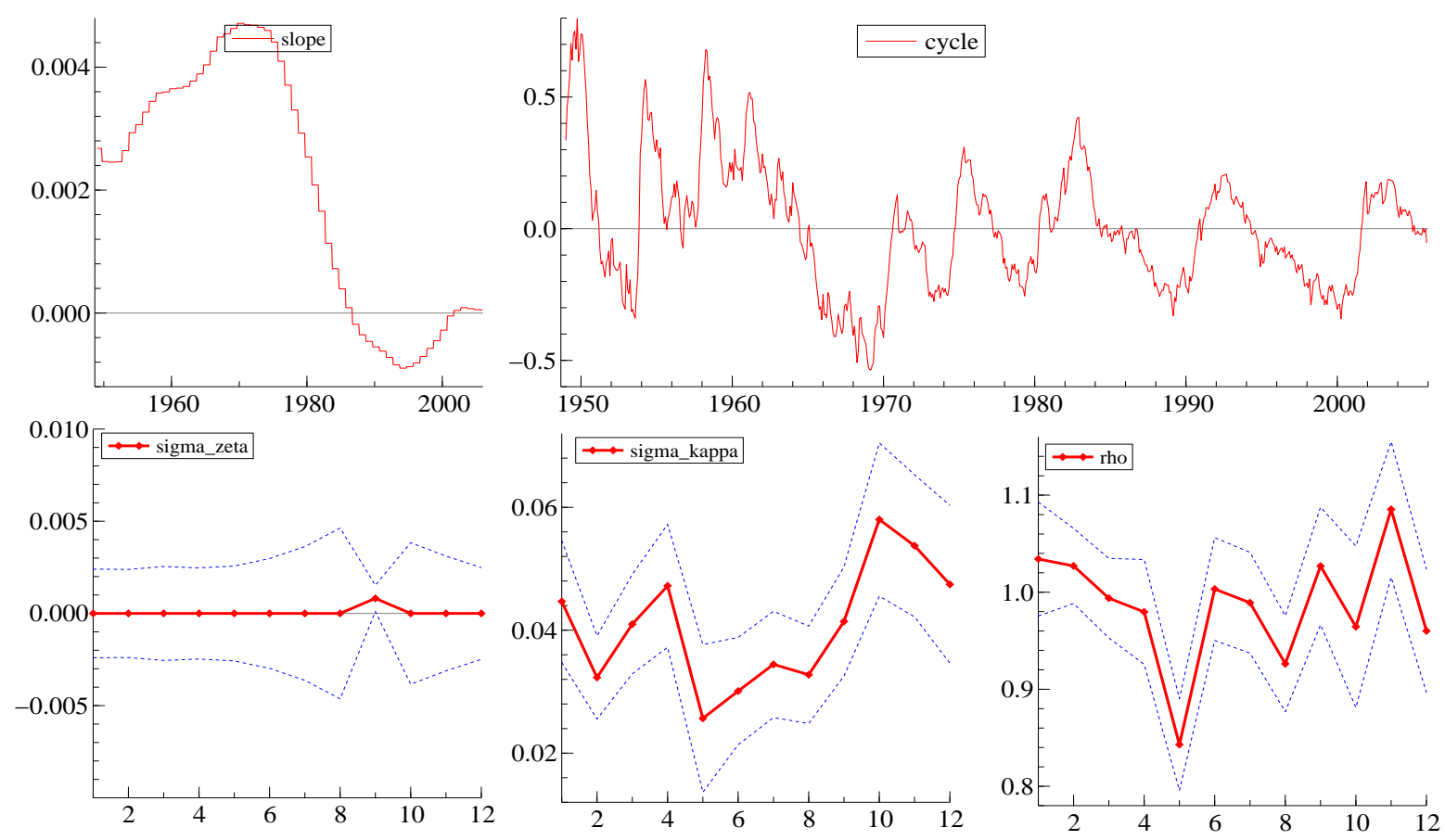

Figure 7: Estimated slope and cycle components (top row) and the corresponding periodic parameters \pm 2 standard errors (bottom row) for the periodic UC model.

Figure 8 provides a closer look on the estimate of the periodic seasonal component $\hat{\gamma}_{t}$. The top panel shows $\hat{\gamma}_{t}$ throughout the whole sample period and the middle panel shows the yearly sub-plots for each month separately. The seasonal pattern for May, June and July turns out to be more volatile than for other months. This is especially clear during the years $1965-1970$ 
as one can see from the top panel. The bottom panel shows how the periodic volatility in the seasonal component is reflected in the standard errors of the seasonal shocks, $\sigma_{\omega, s}$.
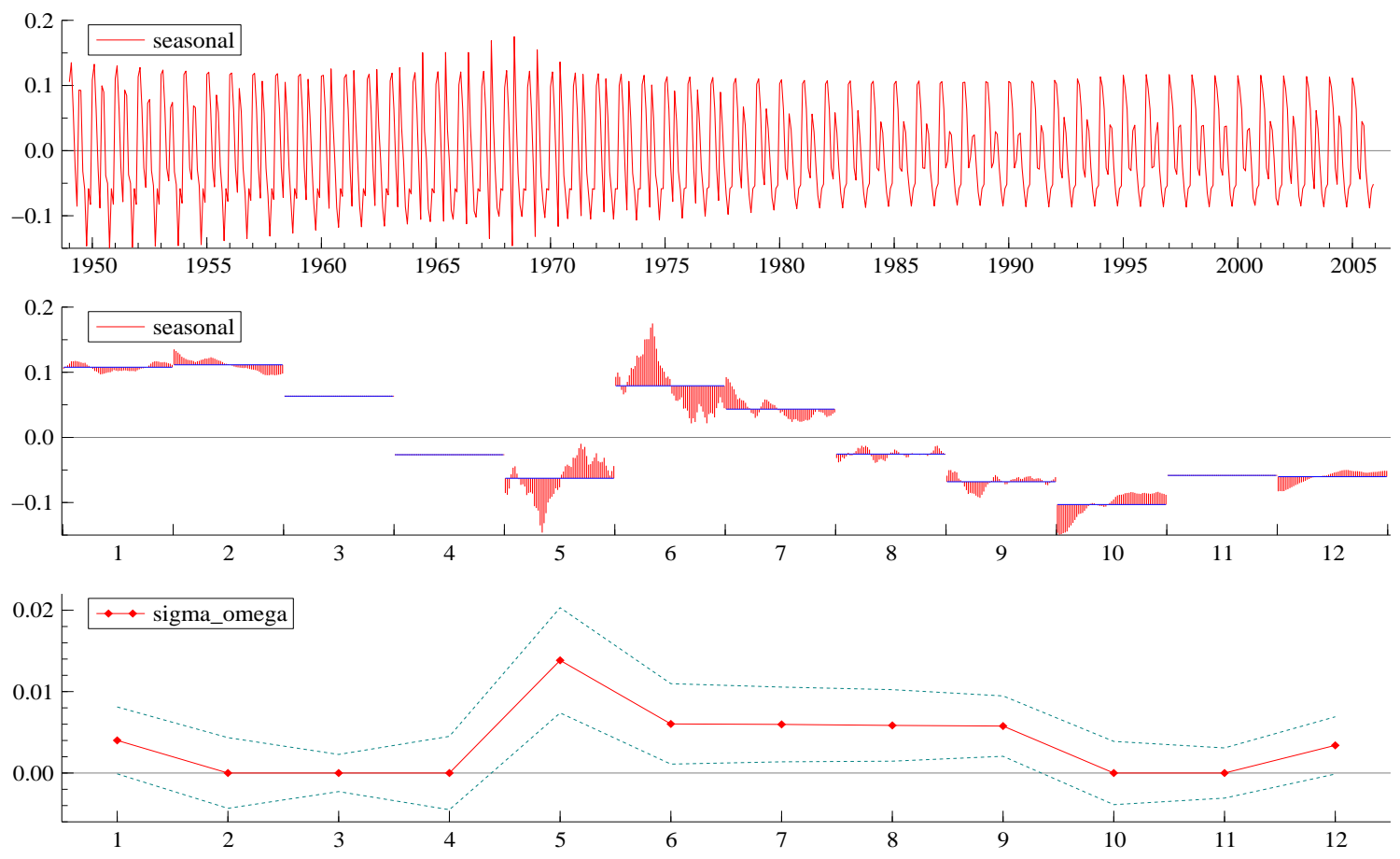

Figure 8: Monthly seasonal component. Top: $\hat{\gamma}_{t}, t=1, \ldots, 696$, Middle: $\hat{\gamma}_{s, t *}, t^{*}=1, \ldots, 58$, $s=1, \ldots, 12$. Bottom: $\hat{\sigma}_{\omega, s} \pm 2$ s.e., $s=1, \ldots, 12$.

Note again that the standard errors of the two other possible types of shocks in the model, $\sigma_{\varepsilon, s}$ and $\sigma_{\eta, s}$, are excluded from the graphs as they are estimated as zero and subsequently fixed. The remaining $73-24=49$ parameters are estimated unrestrictedly.

In order to formally test whether the $\rho$ 's and $\sigma_{\kappa}$ 's are periodic we also estimate the model with additional restrictions. The first set of restrictions imposes that all $\rho$ 's are equal. This results in a more volatile trend estimate and in a shorter average period for the cycle, namely 5.3 years instead of the 10.7 years we obtained for the unrestricted model. The second set of restrictions imposes that all $\sigma_{\kappa}$ 's are equal. This also results in a more volatile trend estimate and in an even shorter cyclical period of 3.2 years. We observe an interesting trade-off between estimates of $\rho$ 's, $\sigma_{\kappa}$ 's and $\lambda$, that is if $\rho$ 's or $\sigma_{\kappa}$ 's are not periodic, we have a large value of $\lambda$ while if $\rho$ 's and $\sigma_{\kappa}$ 's are both periodic, we have a small value of $\lambda$ which results in a longer average length of the cycle.

Table 1 compares the likelihoods of the different periodic and nonperiodic models. The LR-tests for nonperiodicity in the cyclical component are highly significant (compared to the $\chi_{11}^{2}$ critical 
Table 1: Likelihood Ratio tests for periodicity in UC and SARIMA models (sample 1948-2005).

\begin{tabular}{lccccc}
\hline Model & $\log L$ & $p$ & $L R$ & $k$ & $\chi_{k ; 95 \%}^{2}$ \\
\hline$H_{1,1}:$ PUC model & 1116.13 & 49 & & & \\
$H_{0,1}:$ all $\sigma_{\kappa, s}$ equal & 1101.28 & 38 & $29.70^{*}$ & 11 & 19.68 \\
$H_{0,2}:$ all $\rho_{s}$ equal & 1090.69 & 38 & $50.88^{*}$ & 11 & 19.68 \\
$H_{0,3}:$ all PUC parameters are nonperiodic & 1061.28 & 5 & $109.70^{*}$ & 44 & 60.48 \\
& & & & & \\
$H_{1,2}:$ PSARIMA model & 1128.23 & 60 & & & \\
$H_{0,4}:$ all PSARIMA parameters are nonperiodic & 1076.04 & 5 & $104.35^{*}$ & 55 & 73.31 \\
\hline
\end{tabular}

NOTES: $\log L: \log$ likelihood. LR: LR test against $H_{1,1}, H_{1,2}$, respectively. $p$ is the number of estimated parameters. $k$ is the number of restricted parameters. $H_{1,1}$ : PUC model in $(38), \sigma_{\eta, s}^{2}=\sigma_{\varepsilon, s}^{2}=0, s=1, \ldots, 12$. $H_{1,2}$ : PSARIMA model in (39), without term for $\varepsilon_{t-13 .}{ }^{*}: H_{0, i}$ is rejected at $5 \%$ significance level.

value for a significance level $\alpha=0.05)$ which indicates that all $\rho_{s}$ and $\sigma_{\kappa, s}$ are periodic indeed. An additional LR statistic examines the overall periodicity of the periodic UC model, testing whether all variances and damping factors are equal throughout the year against our periodic alternative. The test clearly rejects at $5 \%$ significance level. Table 1 confirms the conclusion from the data analysis and the graphical assessment of the time series decomposition that periodicity is statistically significant in US unemployment series.

The bottom panel of Figure 5 presents graphical diagnostics for the periodic UC model, which can be compared with the equivalent results for the periodic SARIMA model. As we noted earlier for the SARIMA model, we observe that the variance of the one-step-ahead forecast errors is higher between 1948 and 1980. The ACF of the forecast errors shows weak cyclical movements.

To conclude our first periodic unobserved component analysis of US unemployment we compare the likelihoods of the periodic UC and the periodic SARIMA models in Table 1. Thanks to our common state space framework for SARIMA and UC models in terms of the undifferenced series $y_{t}$, the loglikelihoods of the different models can be compared to each other. The loglikelihoods for both periodic models are clearly higher than for their non-periodic counterparts and the differences are significant on the basis of standard asymptotic $\chi^{2}$-tests. The loglikelihood for the periodic SARIMA model is higher than for the periodic UC model, but the latter has a smaller number of parameters. Our periodic SARIMA model in $\S 4.2$ has 60 free parameters, while our periodic UC model only has 49. 


\subsection{Cycle variance moderation in US unemployment}

It appears from Figure 5 that the forecast error variance for US unemployment is much lower after 1980 than before. This structural volatility change is a well documented stylised fact occurring in several US macroeconomic time series, see, e.g., Sensier \& van Dijk (2004) and Kim, Nelson \& Piger (2004). A structural explanation of this decrease in volatility is still a matter of discussion. In monetary economics, the decrease in US unemployment volatility can be associated with a change in monetary policy. Warne \& Vredin (2006) find support for this explanation in a bivariate Structural Vector Autoregressive (SVAR) model for inflation and unemployment, where volatility breaks are captured by endogenous two-regime MarkovSwitching. Primiceri (2005) captures the changing variance in a stochastic volatility specification of a trivariate SVAR, also including an interest rate. Sims \& Zha (2006) analyse a SVAR with six variables switching between nine regimes. All these authors use seasonally adjusted data and find significant changes in US unemployment volatility. As the forecast standard error has decreased by $50 \%$ this is a crucial issue for realistic interval forecasting at the end of our sample.

We take the approach of, i.a., Sensier \& van Dijk (2004) by allowing for breakpoints in the stochastic process governing US unemployment, starting with one breakpoint. By estimating the parameters for two sub-samples, we found that only by varying the $\sigma_{\kappa}$ 's parameters (the shocks of the cycle component), large increases in the likelihood are obtained. Formally, we extend the model for the variances of the cycle component as $\sigma_{\kappa, s, \tau}^{2}$, adding an extra variance factor $\tau$, with

$$
\tau=\left\{\begin{array}{l}
1, \text { for } t \text { in the period from } 1948.1 \text { to } h .12 \\
2, \text { for } t \text { in the period from }(h+1) .1 \text { to } 2005.12
\end{array}\right.
$$

where $h$ is the breakpoint year. The largest increase in the likelihood was found by having the break in the early 1980s, which is in line with results in the literature. The differences in the likelihoods for different breakpoints in the early 1980s are small. We decided to fix the breakpoint in 1982. Warne \& Vredin (2006) found additional variance switches before 1982, but for the purpose of this paper we found one variance break to be satisfactory.

We emphasise that while the cyclical shock volatilities $\sigma_{\kappa, s}$ are distinctly different before and after 1982, the other variances in the model are similar for the two eras. On average, $\sigma_{\kappa}$ was 0.06 in 1948-1981 and 0.03 after 1982. Between the individual $\sigma_{\kappa}$ 's for the different periods, the values of $\sigma_{\kappa}$ 's can differ by a factor 3 . These results agree with those obtained by Kim et al. (2004), who compared univariate unobserved component models with structural variance breaks and Markov Switching. They showed that "the growth rate of aggregate real GDP has been less 
volatile since the early 1980's, and that this volatility reduction is concentrated in the cyclical component of real GDP". As the variance in unemployment is closely related to the variance in GDP growth, it is not surprising to find a similar phenomenon in the unemployment series.

We introduce a similar variance change in the SARIMA model. As we do not distinguish between different variance components in the periodic SARIMA model, we allow for different values of the $\sigma_{\varepsilon, s}$ 's in the two subsamples. The estimated $\sigma_{\varepsilon, s}$ are on average 0.07 before 1982 and 0.03 after 1982, which is in line with the variance estimates for the two unemployment regimes in the model of Warne \& Vredin (2006). The variance moderation has a large effect on the estimates of the long term growth rates in the periodic SARIMA model. While we obtained an overall annual growth rate of $2 \%$ in the homoskedastic model, in the model with variance moderation, the annual growth rate is a mere $0.8 \%$.

Table 2: Estimation results for models with variance moderation in 1982 (sample 1948 - 2005).

\begin{tabular}{lrrrr}
\hline & \multicolumn{2}{c}{ non-periodic models } & \multicolumn{2}{c}{ periodic models } \\
& SARIMA & UC & PSARIMA & PUC \\
\hline $\log L$ & 1142.93 & 1131.04 & 1188.34 & 1188.64 \\
AIC & -3.26 & -3.23 & -3.17 & -3.27 \\
BIC & -3.22 & -3.19 & -2.62 & -2.94 \\
LR & $90.82^{*}$ & $115.20^{*}$ & & \\
$N$ & 1.35 & 0.06 & 0.81 & 1.20 \\
$Q(96)$ & $153.51^{*}$ & $131.54^{*}$ & $177.18^{*}$ & $119.58^{*}$ \\
$Q(144)$ & $236.61^{*}$ & $189.01^{*}$ & $248.05^{*}$ & $173.29^{*}$ \\
$\tilde{p}$ & 6 & 6 & 72 & $50^{\dagger}$ \\
$n^{*} S$ & 696 & 696 & 696 & 696 \\
\hline
\end{tabular}

NOTES: $\log L$ : $\log$-likelihood. AIC: Akaike Information Criterion. BIC: Bayesian Information Criterion. $L R$ is LR test of the nonperiodic model against its periodic counterpart, $N$ is a normality test on the prediction errors; $N \stackrel{a}{\sim} \chi^{2}(2) . Q$ is a Portmanteau autocorrelation test; $Q(l) \stackrel{a}{\sim} \chi^{2}(l-\tilde{p})$ where $l$ is the number of lags and $\tilde{p}$ is the number of parameters. ${ }^{*}$ : the test rejects at a $5 \%$ significance level. ${ }^{\dagger}$ : the number of parameters is calculated as $49+12$ (cycle variance moderation parameters $\left.\sigma_{\kappa, s, 1982}\right)-11$ (zeros in $\left.\sigma_{\zeta, s}\right) \cdot n^{*} S$ is the number data points.

Table 2 reports the loglikelihood values, Akaike's information criteria (AIC), Bayes information criteria (BIC) and tests on the one-step-ahead prediction errors for the models with the variance moderation. By introducing a breakpoint in 1982, heteroskedasticity and nonnormality of the one-step prediction errors have largely disappeared. The loglikelihoods show a dramatic improvement because of the variance moderation. For example, in the periodic UC model we 

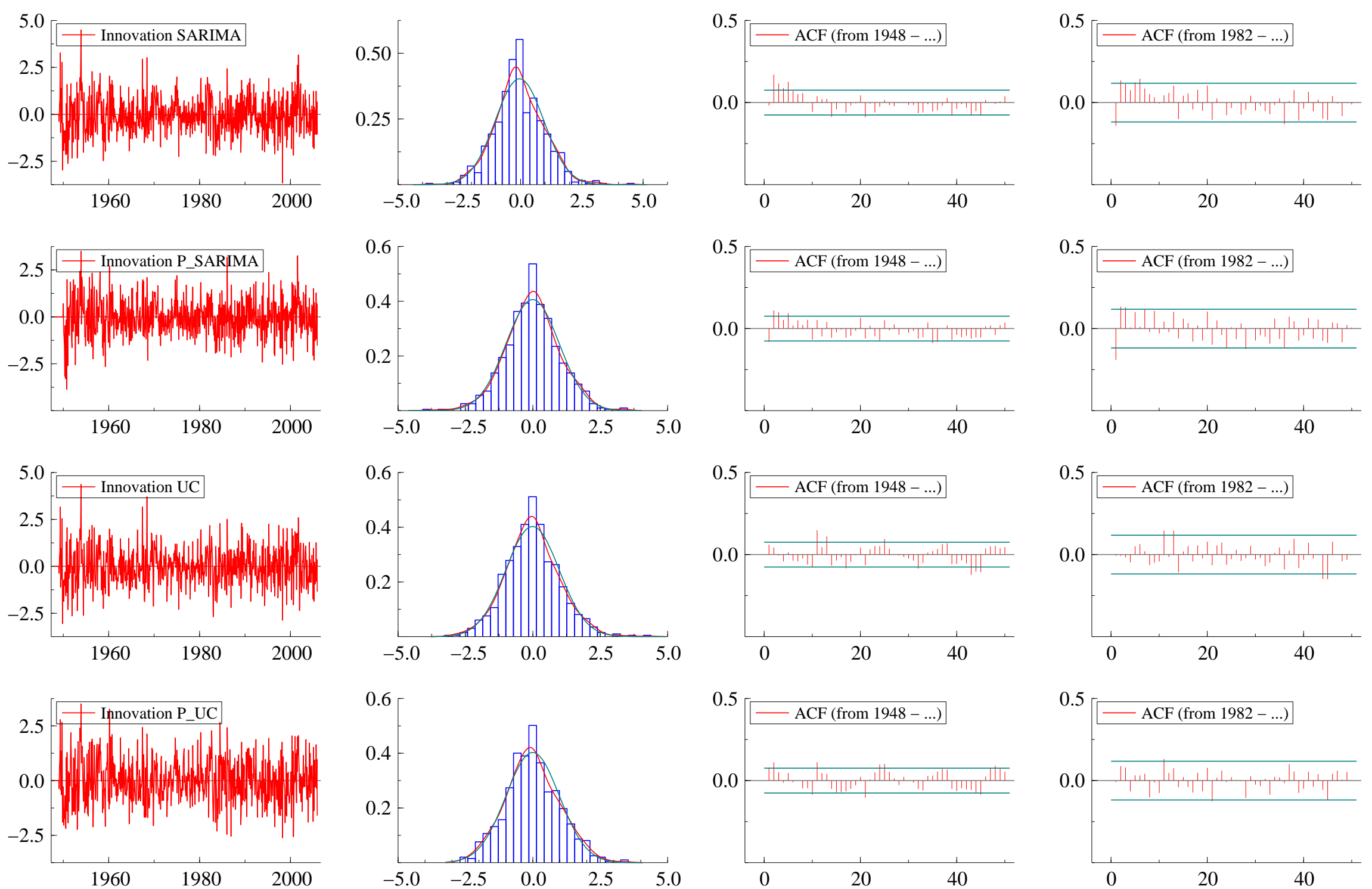

Figure 9: Graphical diagnostics for models reported in Table 1. From left to right: Standardised one-step-ahead prediction errors time series plot, histogram and nonparametric density estimate, sample ACF for the whole sample (from 1948 - 2005) and sample ACF for the second half (from 1982 - 2005) of the series. First row: non-periodic SARIMA model, Second row: periodic SARIMA model, Third row: non-periodic UC model, Fourth row: periodic UC model. 
have an increase in the loglikelihood of 72 , from 1116 to 1188, by introducing twelve extra cycle variance moderation parameters. This increase is highly significant. However, comparing the periodic and nonperiodic SARIMA model on the basis of AIC and BIC, the nonperiodic model is preferred. Between the periodic models themselves, the periodic UC model outperforms the periodic SARIMA based on loglikelihood, AIC, BIC and serial correlation tests for the forecast errors.

Figure 9 presents graphical diagnostics for the whole sample and for the second part of the sample. For forecasting we are primarily interested in the adequacy of the model in the second part of the sample. The diagnostics are now satisfactory. Some serial correlation in the residuals remains, but the cyclical nature of the ACF for the periodic UC forecast errors has disappeared after 1982, see the last row and last column of Figure 9.

Figure 10 shows the changes in the cyclical variances. The most remarkable changes occur in the last three months of the year which were the most volatile months before 1982, turning into relatively tranquil months in the second part of the sample.
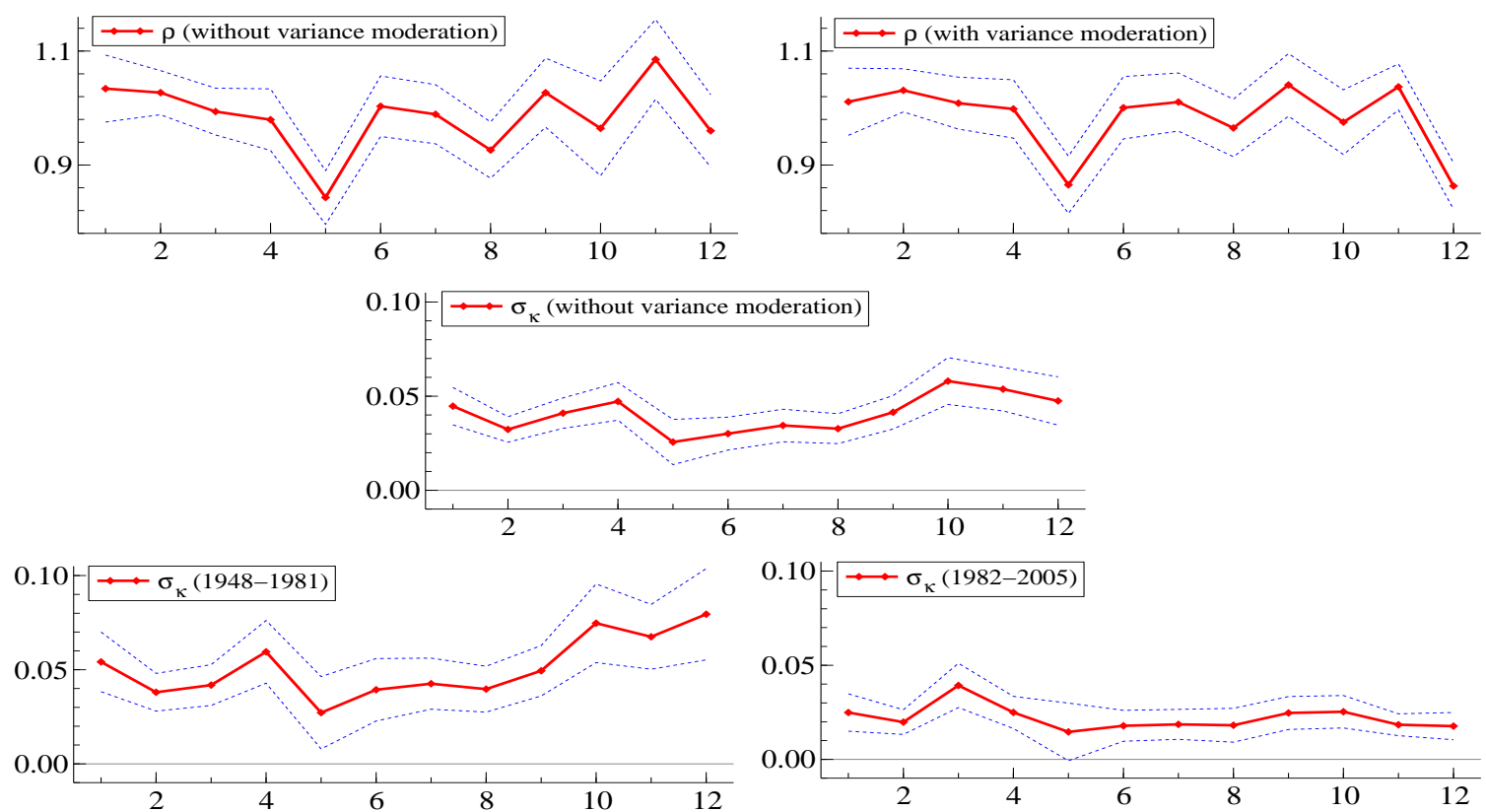

Figure 10: Periodic parameter estimates \pm 2 standard errors for the periodic UC models without and with variance moderation. Top: $\hat{\rho}_{s}$ for model without (left) and with (right) variance moderation. Middle: $\hat{\sigma}_{\kappa, s}$ for model without variance moderation. Bottom: $\hat{\sigma}_{\kappa, s}$ for first and second part of the sample in model with variance moderation. See also Figure 7. 


\subsection{Forecasting weights and forecasting performance}

In the previous subsections we have shown the effects of periodicity on the time series decomposition of US unemployment. This subsection concerns the effect on forecasting. In order to interpret the relative forecasting performance of different models, it is useful to compute the forecasting functions implied by the different specifications. In particular, it is important to see how the weights of past observations in the forecast functions differ from month to month.

Periodic observation weights for $h$-step ahead forecasting are defined similarly as for nonperiodic models, see Harvey \& Koopman (2000), only now different weights apply for different months of the year. Consider the optimal linear one-step ahead forecasting function for the actual series

$$
\hat{y}_{t \mid t-1}=\mathbb{E}\left[y_{t} \mid y_{1}, \ldots, y_{t-1}\right]=w_{1, s, t} y_{t-1}+w_{2, s, t} y_{t-2}+\ldots+w_{t-1, s, t} .
$$

In this notation we have made explicit that the forecasting function with $s=1$ applies when $y_{t}$ falls in month 1 , the forecasting function with $s=2$ applies when $y_{t}$ falls in month 2 , etc. The weights $w_{i, s, t}$ are simply the coefficients of these forecasting functions.

The forecasting weights sum to unity in all models that we discuss in this paper: $\sum_{i=1}^{t-1} w_{i, s, t}=1$. The forecast weight functions of non-periodic models are very similar across different months that are close to each other, especially towards the end of the sample. For periodic models the weight functions are similar for observations exactly one year apart, but the weight functions can be quite different for different months in the same year. The forecasting weights are easy to compute for linear models in state space form. We use the efficient algorithms derived by Koopman \& Harvey (2003). Similar weight functions can be defined for filtered or smoothed trend, cycle and seasonal components of $y_{t}$, but we do not present them in this paper to save space.

Figure 11 shows the weighting patterns for one-step ahead prediction of the observation $y_{2005 . s}$ for the months March, June, September and December 2005, $s=3,6,9,12$. We plot $w_{i, s, t}$ against $-i,-i=-49, \ldots,-1$, for $s=3,6,9,12$. The plots present the $w_{i}$ in reverse order as the weight with the smallest index $i$ correspond to the most recent observation. As expected, the plots show that the prediction of month $t$ depends heavily on the observation of month $t-1$ which is depicted as the last bar of each weight function. Scanning the graph from right to left, we see that the last bar is preceded by small negative weights for the months $t-2$ until $t-11$. The second large and positive bar presents the weight of month $t-12$ from the year before, as is common in seasonal time series models. The first large negative weight occurs for $t-13$, which is common for seasonal time series models with trends as it reflects the difference 
operator. A yearly pattern then repeats itself and damps out. These weight patterns clearly indicate the relevance of the periodic analysis for forecasting, as they are quite distinct for the different months of the year.
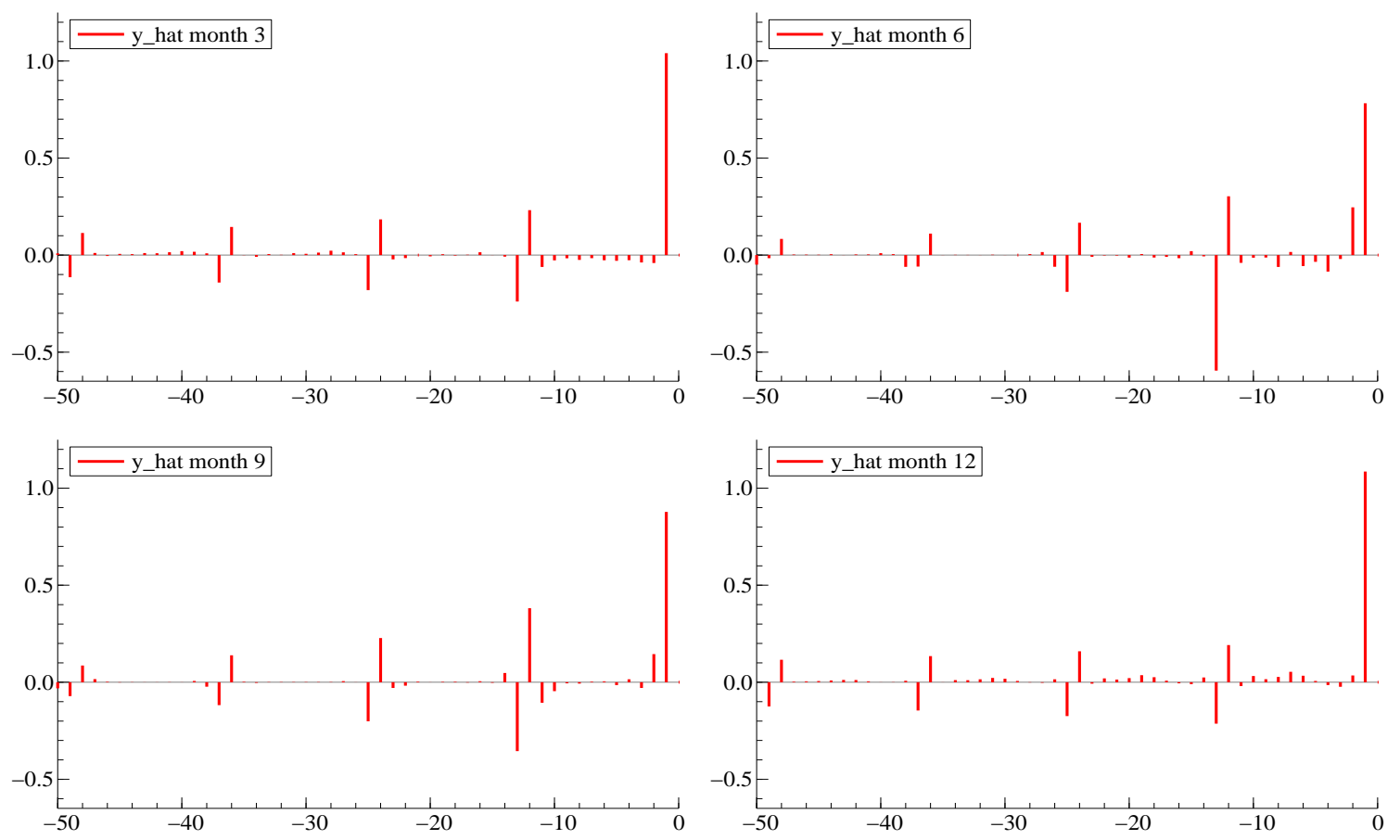

Figure 11: Observation weights of $y_{t-i},-i=-50 \ldots,-1$ for one-step ahead prediction of the observation $\left(y_{t}\right)$ for the months March, June, September and December 2005.

Figure 12 shows the 1 to 8 -month ahead forecasts for 2006.1 - 2006.8 together with their $67 \%$ confidence interval for all models of Table 2 and compares them with actual values. The forecasts are quite satisfactory. The top row presents the forecasts of the non-periodic models and the bottom row depicts the forecasts of the periodic models. The left column is for the SARIMA models and the right column is for the UC models. In general, the standard errors of the periodic models are smaller than the non-periodic ones and therefore the forecasts of the periodic models are more accurate than those of non-periodic ones.

Comparing the two periodic models, the forecasts look similar for the first five months which are somewhat higher than the realised values. The forecast of the periodic UC model is almost perfect for June 2006 but becomes less accurate in July and August. The reverse holds for the forecasts of the periodic SARIMA model which are higher than the realised unemployment in June but quite precise in July and August. In sum, both periodic models produce realistic outof-sample point- and interval forecasts without significant differences between the two models. 

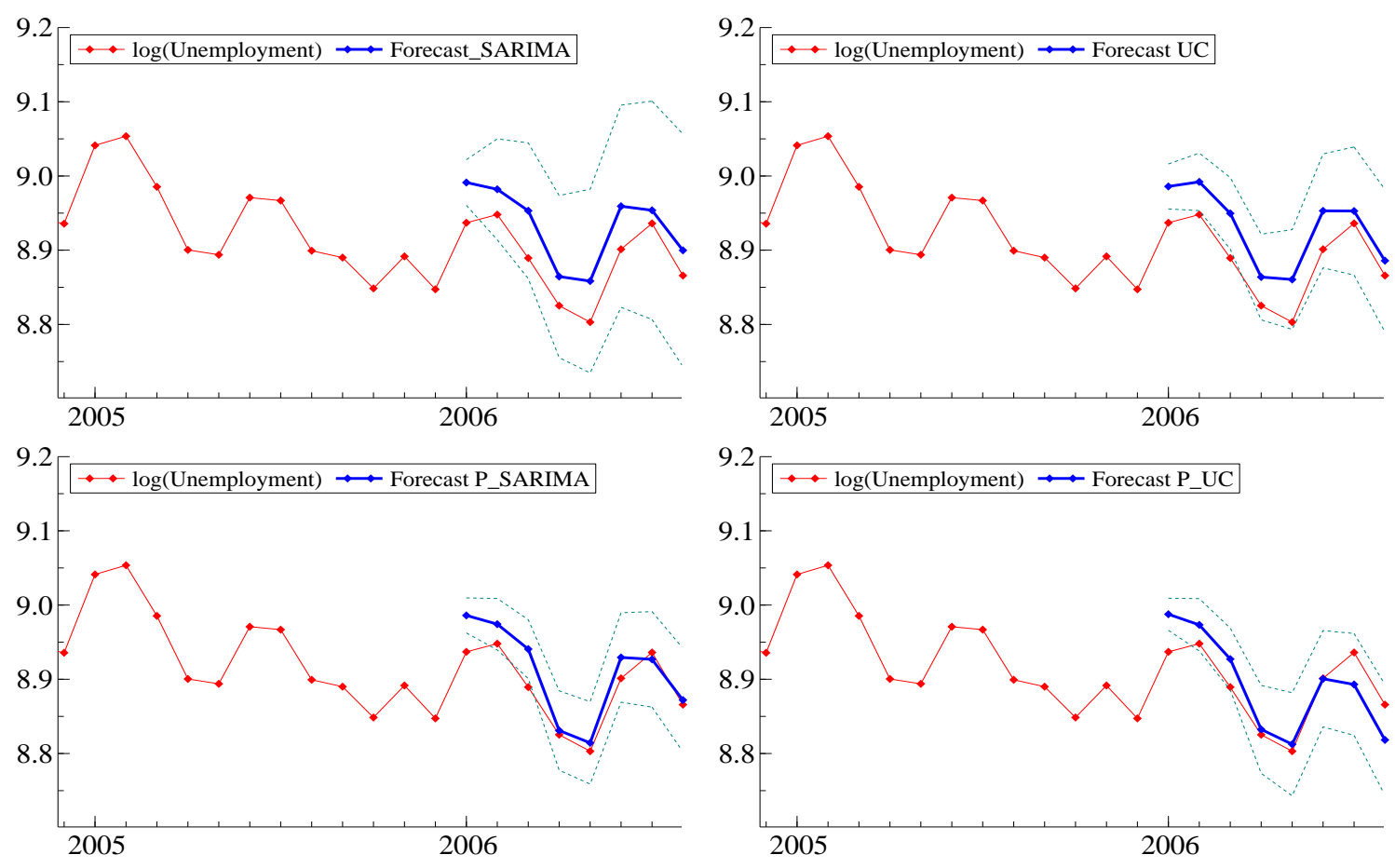

Figure 12: Forecasted series with standard errors over year 2005 using non-periodic (top panel) and periodic (bottom panel) models.

\section{Summary and Conclusion}

The primary aim of the paper was to develop appropriate methods and algorithms for estimation, decomposition, diagnostic checking and forecasting. We succeed to present a comprehensive analysis of univariate periodic unobserved component modelling where we distinguish four stochastic components: a (smooth) stochastic trend, a seasonal, a cycle and an irregular. In addressing to the discussion about identification problem, we find that our periodic unobserved component models are identified for $S>2$, where $S$ is the number of observations per year. For $S=2$, a parameter restriction on the seasonal component is needed. We also have derived two equivalent state space forms for the periodic unobserved components model, a univariate timevarying and a multivariate constant-parameter formulation. Both can be used for estimation, decomposition and forecasting.

Estimation of the unknown parameters is feasible despite the possibly large number of parameters. Estimates are obtained by the exact maximum likelihood method with diffuse initialisation of the nonstationary components in the model. We develop a related new exact estimation method for periodic SARIMA models, which does not require a priori differencing of the data. In our application to monthly postwar US unemployment, we discover periodicity in all pa- 
rameters, especially in the cyclical component. Periodic models fit the data better than the non-periodic ones in terms of loglikelihood and out-of-sample forecasts. As the variances of cyclical unemployment decreased markedly in the second part of our sample we successfully incorporate a variance moderation into our model. Both the level and the periodic pattern of the cyclical variance changed over time. Our trend estimate of US unemployment shows a clear periodic pattern with the largest conditional variance in October of each year. The observation weights for forecasting also show some marked differences in the forecasting functions for different months in the same year.

The model can be extended in several ways. In shorter samples, placing extra restrictions on the intra-year pattern of the periodic coefficients may be needed, as is common in geophysical applications of periodic models. Another extension is allowing for more than one independent trend component to bridge the gap with independent periodic models which specify independent trends for different months of the year. In that case non-trivial identification problems for seasonal adjustment arise, see the discussion in $\S 1$. Extensions of the statistical analysis are also possible within our framework. Following Busetti \& Harvey (2003), stationarity tests for the periodic components in the model can be developed.

Different nonlinear model extensions can be considered, but they require the use of nonlinear or non-Gaussian state space formulations that increase the computational complexity. Further research is planned in this direction. Finally, the periodic approach can be extended to multivariate UC time series models. For US unemployment, an extension with monetary variables leads to models of economic interest. However, it is an empirical question whether such extensions will result in better decompositions or more accurate forecasts.

\section{Appendices}

\section{A Moments and identification local linear trend model for $S=2$}

In this appendix we extend the analysis of $\S 3.3$, allowing for a stochastic slope $\beta_{t}$. We consider a univariate periodic local linear trend (PLLT) model for half-yearly data with periodic variances for the disturbances of the trend, slope, seasonal and irregular components

$$
\begin{array}{rlrl}
y_{t} & =\mu_{t}+\gamma_{t}+\varepsilon_{t}, & \varepsilon_{t} \sim \operatorname{NID}\left(0, \sigma_{\varepsilon, s}^{2}\right), \\
\mu_{t+1}=\mu_{t}+\beta_{t}+\eta_{t}, & \eta_{t} \sim \operatorname{NID}\left(0, \sigma_{\eta, s}^{2}\right), \\
\beta_{t+1}=\beta_{t}+\zeta_{t}, & \zeta_{t} \sim \operatorname{NID}\left(0, \sigma_{\zeta, s}^{2}\right), \\
\gamma_{t+1}=-\gamma_{t}+\omega_{t}, & \omega_{t} \sim \operatorname{NID}\left(0, \sigma_{\omega, s}^{2}\right),
\end{array}
$$

for $t=1, S+1,2 S+1, \ldots$, where $s=1, \ldots, S$ and $S=2$. As before, a high $\sigma_{\varepsilon, 1}^{2}$ is associated with a high variance for $y_{t}$ in period 1 , but high values in $\sigma_{\eta, 1}^{2}, \sigma_{\zeta, 1}^{2}$ and $\sigma_{\omega, 1}^{2}$ are associated with 
a high variance in the next period.

The stationary form of the periodic local linear trend model is given by

$$
\begin{aligned}
\Delta \Delta_{2} y_{t} & =\eta_{t-1}-\eta_{t-3}+\zeta_{t-2}+\zeta_{t-3}+\omega_{t-1}-2 \omega_{t-2}+\omega_{t-3}+\varepsilon_{t}-\varepsilon_{t-1}-\varepsilon_{t-2}+\varepsilon_{t-3} \\
\Delta \Delta_{2} y_{t+1} & =\eta_{t}-\eta_{t-2}+\zeta_{t-1}+\zeta_{t-2}+\omega_{t}-2 \omega_{t-1}+\omega_{t-2}+\varepsilon_{t+1}-\varepsilon_{t}-\varepsilon_{t-1}+\varepsilon_{t-2}
\end{aligned}
$$

for $t=1, S+1,2 S+1, \ldots$, where $\Delta \Delta_{S} y_{t}=(1-L)\left(1-L^{S}\right) y_{t}$. The autocovariance function of the above model for $t=1, S+1,2 S+1, \ldots$ has a $\operatorname{VMA}(2)$ structure,

$$
\begin{aligned}
& \Gamma_{0}=\mathbb{E}\left[\left(\begin{array}{c}
\Delta \Delta_{2} y_{t} \\
\Delta \Delta_{2} y_{t+1}
\end{array}\right)\left(\begin{array}{c}
\Delta \Delta_{2} y_{t} \\
\Delta \Delta_{2} y_{t+1}
\end{array}\right)^{\prime}\right]=\left(\begin{array}{cc}
\gamma_{11,0} & \gamma_{12,0} \\
\gamma_{21,0} & \gamma_{22,0}
\end{array}\right) \\
& \Gamma_{1}=\mathbb{E}\left[\left(\begin{array}{c}
\Delta \Delta_{2} y_{t} \\
\Delta \Delta_{2} y_{t+1}
\end{array}\right)\left(\begin{array}{c}
\Delta \Delta_{2} y_{t-2} \\
\Delta \Delta_{2} y_{t-1}
\end{array}\right)^{\prime}\right]=\left(\begin{array}{cc}
\gamma_{11,1} & \gamma_{12,1} \\
\gamma_{21,1} & \gamma_{22,1}
\end{array}\right) \\
& \Gamma_{2}=\mathbb{E}\left[\left(\begin{array}{c}
\Delta \Delta_{2} y_{t} \\
\Delta \Delta_{2} y_{t+1}
\end{array}\right)\left(\begin{array}{c}
\Delta \Delta_{2} y_{t-4} \\
\Delta \Delta_{2} y_{t-3}
\end{array}\right)^{\prime}\right]=\left(\begin{array}{cc}
0 & \gamma_{12,2} \\
0 & 0
\end{array}\right) \\
& \Gamma_{j}=\mathbb{E}\left[\left(\begin{array}{c}
\Delta_{2} y_{t} \\
\Delta \Delta_{2} y_{t+1}
\end{array}\right)\left(\begin{array}{l}
\Delta \Delta_{2} y_{t-2 j} \\
\Delta_{2} y_{t+1-2 j}
\end{array}\right)^{\prime}\right]=0
\end{aligned}
$$

where

$$
\begin{aligned}
\gamma_{11,0} & =2 \sigma_{\eta, 2}^{2}+\sum_{i=1}^{2} \sigma_{\zeta, i}^{2}+4 \sigma_{\omega, 1}^{2}+2 \sigma_{\omega, 2}^{2}+2 \sum_{i=1}^{2} \sigma_{\varepsilon, i}^{2} \\
\gamma_{12,0} & =\sigma_{\zeta, 1}^{2}-2 \sum_{i=1}^{2} \sigma_{\omega, i}^{2}-2 \sigma_{\varepsilon, 1}^{2}+\sigma_{\varepsilon, 2}^{2} \\
\gamma_{21,0} & =\gamma_{12,0} \\
\gamma_{22,0} & =2 \sigma_{\eta, 1}^{2}+\sum_{i=1}^{2} \sigma_{\zeta, i}^{2}+2 \sigma_{\omega, 1}^{2}+4 \sigma_{\omega, 2}^{2}+2 \sum_{i=1}^{2} \sigma_{\varepsilon, i}^{2} \\
\gamma_{11,1} & =-\sigma_{\eta, 2}^{2}+\sigma_{\omega, 2}^{2}-\sum_{i=1}^{2} \sigma_{\varepsilon, i}^{2} \\
\gamma_{12,1} & =\sigma_{\zeta, 2}^{2}-2 \sum_{i=1}^{2} \sigma_{\omega, i}^{2}+\sigma_{\varepsilon, 1}^{2}-2 \sigma_{\varepsilon, 2}^{2} \\
\gamma_{21,1} & =\sigma_{\varepsilon, 1}^{2} \\
\gamma_{22,1} & =-\sigma_{\eta, 1}^{2}+\sigma_{\omega, 1}^{2}-\sum_{i=1}^{2} \sigma_{\varepsilon, i}^{2} \\
\gamma_{12,2} & =\sigma_{\varepsilon, 2}^{2}
\end{aligned}
$$

from which we obtain 8 linear equations with 8 unknown parameters. The above equations can be rewritten in a matrix equation form, $A \varphi=\Gamma$, where the matrix $A$ represents the linear equations, $\varphi$ contains the vector of unknown parameters and $\Gamma$ consists of different elements of $\Gamma_{0}, \Gamma_{1}$ and $\Gamma_{2}$. The rank of matrix $A$ is 7 , one less than the number of unknowns, which means that one restriction is needed to identify the model. Natural restrictions have the following forms:

$$
\sigma_{\eta, 1}^{2}=\sigma_{\eta, 2}^{2}=\sigma_{\eta}^{2} \quad \text { or } \quad \sigma_{\omega, 1}^{2}=\sigma_{\omega, 2}^{2}=\sigma_{\omega}^{2} \quad \text { or } \quad \sigma_{\omega, 2}^{2}=\sigma_{\eta, 1}^{2}-\sigma_{\eta, 2}^{2}+\sigma_{\omega, 1}^{2}
$$

The loglikelihood of the estimated model is exactly the same for each of these three restrictions. 


\section{B Moments and identification basic structural model for $S=3$}

This appendix extends the analysis of $\S 3.3$ to $S=3$. The stationary form of the periodic BSM model in equation (6) for $t=1, S+1,2 S+1, \ldots$ with $S=3$ is given by

$$
\begin{aligned}
\Delta_{3} y_{t} & =\eta_{t-3}+\eta_{t-2}+\eta_{t-1}-\omega_{t-2}+\omega_{t-1}+\varepsilon_{t}-\varepsilon_{t-3}, \\
\Delta_{3} y_{t+1} & =\eta_{t-2}+\eta_{t-1}+\eta_{t}-\omega_{t-1}+\omega_{t}+\varepsilon_{t+1}-\varepsilon_{t-2}, \\
\Delta_{3} y_{t+2} & =\eta_{t-1}+\eta_{t}+\eta_{t+1}-\omega_{t}+\omega_{t+1}+\varepsilon_{t+2}-\varepsilon_{t-1},
\end{aligned}
$$

The autocovariance function for $t=1, S+1,2 S+1, \ldots$ is given by

$$
\begin{aligned}
& \Gamma_{0}=\mathbb{E}\left[\left(\begin{array}{c}
\Delta_{3} y_{t} \\
\Delta_{3} y_{t+1} \\
\Delta_{3} y_{t+2}
\end{array}\right)\left(\begin{array}{c}
\Delta_{3} y_{t} \\
\Delta_{3} y_{t+1} \\
\Delta_{3} y_{t+2}
\end{array}\right)^{\prime}\right] \\
& =\left[\begin{array}{ccc}
\sum_{i=1}^{3} \sigma_{\eta, i}^{2}+\sigma_{\omega, 2}^{2}+\sigma_{\omega, 3}^{2}+2 \sigma_{\varepsilon, 1}^{2} & \sigma_{\eta, 2}^{2}+\sigma_{\eta, 3}^{2}-\sigma_{\omega, 3}^{2} & \sigma_{\eta, 3}^{2} \\
\sigma_{\eta, 2}^{2}+\sigma_{\eta, 3}^{2}-\sigma_{\omega, 3}^{2} & \sum_{i=1}^{3} \sigma_{\eta, i}^{2}+\sigma_{\omega, 1}^{2}+\sigma_{\omega, 3}^{2}+2 \sigma_{\varepsilon, 2}^{2} & \sigma_{\eta, 1}^{2}+\sigma_{\eta, 3}^{2}-\sigma_{\omega, 1}^{2} \\
\sigma_{\eta, 3}^{2} & \sigma_{\eta, 1}^{2}+\sigma_{\eta, 3}^{2}-\sigma_{\omega, 1}^{2} & \sum_{i=1}^{3} \sigma_{\eta, i}^{2}+\sigma_{\omega, 1}^{2}+\sigma_{\omega, 2}^{2}+2 \sigma_{\varepsilon, 3}^{2}
\end{array}\right] \\
& \Gamma_{1}=\mathbb{E}\left[\left(\begin{array}{c}
\Delta_{3} y_{t} \\
\Delta_{3} y_{t+1} \\
\Delta_{3} y_{t+2}
\end{array}\right)\left(\begin{array}{c}
\Delta_{3} y_{t-3} \\
\Delta_{3} y_{t-2} \\
\Delta_{3} y_{t-1}
\end{array}\right)^{\prime}\right]=\left[\begin{array}{ccc}
-\sigma_{\varepsilon, 1}^{2} & \sigma_{\eta, 1}^{2} & \sigma_{\eta, 1}^{2}+\sigma_{\eta, 2}^{2}-\sigma_{\omega, 2}^{2} \\
0 & -\sigma_{\varepsilon, 2}^{2} & \sigma_{\eta, 2}^{2} \\
0 & 0 & -\sigma_{\varepsilon, 3}^{2}
\end{array}\right] \\
& \Gamma_{j}=\mathbb{E}\left[\left(\begin{array}{c}
\Delta_{3} y_{t} \\
\Delta_{3} y_{t+1} \\
\Delta_{3} y_{t+2}
\end{array}\right)\left(\begin{array}{c}
\Delta_{3} y_{t-3 j} \\
\Delta_{3} y_{t+1-3 j} \\
\Delta_{3} y_{t+2-3 j}
\end{array}\right)^{\prime}\right]=0 \quad \text { for } j \geq 2
\end{aligned}
$$

Identifiability can be shown by solving the following system of equations

$$
\left[\begin{array}{ccccccccc}
1 & 1 & 1 & 0 & 1 & 1 & 2 & 0 & 0 \\
1 & 1 & 1 & 1 & 0 & 1 & 0 & 2 & 0 \\
1 & 1 & 1 & 1 & 1 & 0 & 0 & 0 & 2 \\
1 & 0 & 1 & -1 & 0 & 0 & 0 & 0 & 0 \\
1 & 1 & 0 & 0 & -1 & 0 & 0 & 0 & 0 \\
0 & 1 & 1 & 0 & 0 & -1 & 0 & 0 & 0 \\
1 & 0 & 0 & 0 & 0 & 0 & 0 & 0 & 0 \\
0 & 1 & 0 & 0 & 0 & 0 & 0 & 0 & 0 \\
0 & 0 & 1 & 0 & 0 & 0 & 0 & 0 & 0 \\
0 & 0 & 0 & 0 & 0 & 0 & -1 & 0 & 0 \\
0 & 0 & 0 & 0 & 0 & 0 & 0 & -1 & 0 \\
0 & 0 & 0 & 0 & 0 & 0 & 0 & 0 & -1
\end{array}\right]\left(\begin{array}{c}
\sigma_{\eta, 1}^{2} \\
\sigma_{\eta, 2}^{2} \\
\sigma_{\eta, 3}^{2} \\
\sigma_{\omega, 1}^{2} \\
\sigma_{\omega, 2}^{2} \\
\sigma_{\omega, 3}^{2} \\
\sigma_{\varepsilon, 1}^{2} \\
\sigma_{\varepsilon, 2}^{2} \\
\sigma_{\varepsilon, 3}^{2}
\end{array}\right)=\left[\begin{array}{c}
\Gamma_{0}(1,1) \\
\Gamma_{0}(2,2) \\
\Gamma_{0}(3,3) \\
\Gamma_{0}(3,2) \\
\Gamma_{1}(1,3) \\
\Gamma_{0}(2,1) \\
\Gamma_{1}(1,2) \\
\Gamma_{1}(2,3) \\
\Gamma_{0}(3,1) \\
\Gamma_{1}(1,1) \\
\Gamma_{1}(2,2) \\
\Gamma_{1}(3,3)
\end{array}\right],
$$


where the vector on the right hand side consists of the different nonzero elements of $\Gamma_{0}$ and $\Gamma_{1}$. The matrix on the left hand side has a full column rank resulting in identifiability. The system is easily solved for $\sigma_{\varepsilon, s}^{2}, \sigma_{\omega, s}^{2}$ and $\sigma_{\eta, s}^{2}$, respectively.

\section{Sample periodic correlations}

Sample periodic correlations have been defined by, i.a., McLeod (1994). In this appendix we discuss the definition and computation that we use in $\S 4.1$. We compute the sample periodic correlation as follows. First, we consider the separate series in $\left\{y_{t^{*}}^{*}\right\}$ where

$$
\left\{y_{t^{*}}^{*}\right\}=\left(\begin{array}{llll}
y_{1, t^{*}} & y_{2, t^{*}} & \cdots & y_{S, t^{*}}
\end{array}\right)^{\prime}
$$

and standardise the series $y_{s, t^{*}}$ separately by subtracting the periodic means and by dividing by the periodic standard deviations to get $\left\{\tilde{y}_{t^{*}}\right\}$, where

$$
\left\{\tilde{y}_{t^{*}}\right\}=\left(\begin{array}{llll}
\tilde{y}_{1, t^{*}} & \tilde{y}_{2, t^{*}} & \cdots & \tilde{y}_{S, t^{*}}
\end{array}\right)^{\prime}
$$

for $s=1, \ldots, S, t^{*}=1,2, \ldots$, where $t^{*}$ is the index in years. If we associate $\tilde{y}_{1, t^{*}}$ with $\tilde{y}_{t}$ (series $\tilde{y}$ at time $t), y_{2, t^{*}}$ with $\tilde{y}_{t+1}$ (series $\tilde{y}$ at time $\left.t+1\right), \ldots$, and $y_{S, t^{*}}$ with $\tilde{y}_{t+S-1}$ (series $\tilde{y}$ at time $t+S-1)$, we can also view the standardised series as

$$
\left\{\tilde{y}_{t^{*}}\right\}=\left(\begin{array}{llll}
\tilde{y}_{t} & \tilde{y}_{t+1} & \cdots & \tilde{y}_{t+S-1}
\end{array}\right)^{\prime}, \quad \text { for } t=1, S+1,2 S+1, \ldots
$$

Next, consider all the covariances between the standardised subseries, $\left\{\tilde{y}_{t^{*}}\right\}$, and their lags $\left\{\tilde{y}_{t^{*}-j}\right\}$, where

$$
\begin{aligned}
\left\{\tilde{y}_{t^{*}-j}\right\} & =\left(\begin{array}{llll}
\tilde{y}_{1, t^{*}-j} & \tilde{y}_{2, t^{*}-j} & \cdots & \tilde{y}_{S, t^{*}-j}
\end{array}\right)^{\prime} \\
& =\left(\begin{array}{llll}
\tilde{y}_{t-j S} & \tilde{y}_{t+1-j S} & \cdots & \tilde{y}_{t+S-1-j S}
\end{array}\right)^{\prime}, \quad \text { for } t=1, S+1,2 S+1, \ldots
\end{aligned}
$$

For $S=2$ the periodic correlations $\gamma_{i, s}, i=1,2, \ldots, s=1, \ldots, S$, are selected from the multivariate correlation matrices of $\tilde{y}_{t^{*}}$ as follows:

$$
\begin{aligned}
\mathbb{E}\left[\left(\begin{array}{c}
\tilde{y}_{1, t^{*}} \\
\tilde{y}_{2, t^{*}}
\end{array}\right)\left(\begin{array}{c}
\tilde{y}_{1, t^{*}} \\
\tilde{y}_{2, t^{*}}
\end{array}\right)^{\prime}\right] & =\left(\begin{array}{cc}
1 & \gamma_{1,-1} \\
\gamma_{2,1} & 1
\end{array}\right) \\
\mathbb{E}\left[\left(\begin{array}{c}
\tilde{y}_{1, t^{*}} \\
\tilde{y}_{2, t^{*}}
\end{array}\right)\left(\begin{array}{c}
\tilde{y}_{1, t^{*}-j} \\
\tilde{y}_{2, t^{*}-j}
\end{array}\right)^{\prime}\right] & =\left(\begin{array}{cc}
\gamma_{1,2 j} & \gamma_{1,2 j+1} \\
\gamma_{2,2 j+1} & \gamma_{2,2 j}
\end{array}\right), \quad j=1,2, \ldots
\end{aligned}
$$

The sample correlations $c_{i, s}, i=1,2, \ldots,(n-1) S, s=1, \ldots, S$ are the sample versions of the population correlations $\gamma_{i, s}$ defined above, without degrees of freedom corrections. For $S>2$ the computation is analogous. In $\S 4.1$, where $S=12$, we report the $c_{i, s}, i=1, \ldots, 120$ and $s=3,6,9,12$. 


\section{Periodic SARIMA model in state space representation}

In this appendix we discuss the state space representation of the periodic SARIMA model of $\S 4.2$. The seasonally differenced $y_{t}^{\dagger}=\Delta_{S} y_{t}$ series follows a simplified periodic $\operatorname{SARMA}(2,0,1)(0,0,1)_{S}$ process with a time-varying-mean, which we write as

$$
y_{t}^{\dagger}=\beta_{t}+\phi_{1, t}\left(y_{t-1}^{\dagger}-\beta_{t}\right)+\phi_{2, t}\left(y_{t-2}^{\dagger}-\beta_{t}\right)+\varepsilon_{t}+\theta_{1, t} \varepsilon_{t-1}+\Theta_{1, t} \varepsilon_{t-S},
$$

with $\varepsilon_{t} \sim N\left(0, \sigma_{\varepsilon, t}^{2}\right)$, where $\beta_{t}, \phi_{1, t}, \phi_{2, t}, \theta_{1, t}, \Theta_{1, t}$ and $\sigma_{\varepsilon, t}^{2}$ are coefficients that vary deterministically across the $S$ different periods of the year. To keep the model as simple as possible, yet adequate enough for log US unemployment, the term $\theta_{1, t} \Theta_{1, t} \varepsilon_{t-(S+1)}$ is omitted. In order to make the likelihood for the periodic SARIMA model (50) comparable with the periodic UC model we rewrite the model in terms of the levels, $y_{t}$, as

$$
y_{t}=\beta_{t}+y_{t-S}+y_{t}^{\ddagger}
$$

where $y_{t}^{\ddagger}=y_{t}^{\dagger}-\beta_{t}=\Delta_{S} y_{t}-\beta_{t}=y_{t}-y_{t-S}-\beta_{t}$ for $t=1,2, \ldots$ This is also an appropriate form to obtain the out-of-sample forecasts for $y_{t}$ and their corresponding standard errors without additional algebra. The elements of the state space form (16)-(17) are given as follows. The state vector $\alpha_{t}$ is defined as

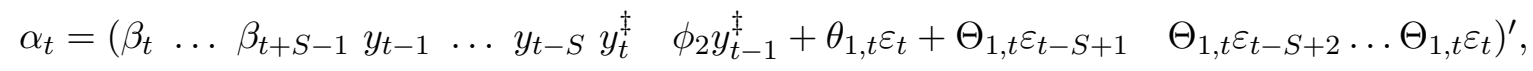
with corresponding disturbance vector given by

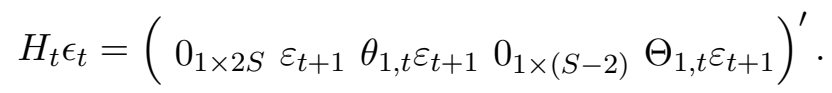

The transition matrix $T_{t}$ is therefore $(3 S+1) \times(3 S+1)$ and $T_{t}$ and $Z_{t}$ are given by

$$
\begin{aligned}
& T_{t}=\left[\begin{array}{cc}
T_{a} & 0_{S \times(2 S+1)} \\
T_{b} & T_{c} \\
0_{(S+1) \times S} & T_{d}
\end{array}\right], \quad Z_{t}=\left(\begin{array}{ll}
1 & \left.0_{1 \times 3 S}\right)
\end{array}\right]\left[\begin{array}{ll}
T_{b} & T_{c}
\end{array}\right], \\
& T_{a}=\left[\begin{array}{cc}
0 & I_{S-1} \\
1 & 0_{1 \times(S-1)}
\end{array}\right], \quad T_{c}=\left[\begin{array}{cccc}
0_{1 \times(S-1)} & 1 & 1 & 0_{1 \times S} \\
I_{S-1} & 0_{(S-1) \times 1} & 0_{(S-1) \times 1} & 0_{(S-1) \times S}
\end{array}\right], \\
& T_{b}=\left[\begin{array}{cc}
1 & 0_{1 \times(S-1)} \\
0_{(S-1) \times 1} & 0_{(S-1) \times(S-1)}
\end{array}\right], \quad T_{d}=\left[\begin{array}{cccc}
0_{2 \times S} & \phi_{1, t} & I_{2} & 0_{2 \times S-2} \\
& \phi_{2, t} & & \\
0_{(S-2) \times S} & 0_{(S-2) \times 1} & 0_{(S-2) \times 2} & I_{(S-2)} \\
0_{1 \times S} & 0 & 0_{1 \times 2} & 0_{1 \times(S-2)}
\end{array}\right] \text {. }
\end{aligned}
$$


where $0_{r \times c}$ denotes a zero matrix with $r$ rows and $c$ columns and where $I_{r}$ denotes an identity matrix of dimension $r . H_{t}$ is defined as

$$
H_{t}=\left(0_{1 \times 2 S} \sigma_{\varepsilon} \theta_{1, t} \sigma_{\varepsilon} 0_{1 \times(S-2)} \Theta_{1, t} \sigma_{\varepsilon}\right)^{\prime},
$$

whereas $G_{t}=0$.

Finally, $a=\mathbb{E}\left(\alpha_{1}\right)=0_{(3 S+1) \times 1}$ and the variance matrix of the initial state is given by

$$
P_{0}=\left[\begin{array}{cc}
\kappa I_{2 S} & 0_{2 S \times(S+1)} \\
0_{(S+1) \times 2 S} & P_{*}
\end{array}\right],
$$

where the initial variance matrices of the growth rates $\beta_{t}$ and the levels $y_{t}$ are diffuse: $\kappa \rightarrow \infty$. It remains to define the variance matrix of the stationary elements of the state, $P_{*}$, where $\operatorname{Var}\left(y_{1}^{\ddagger}\right)$ is the nontrivial term. A simple analytical solution for the unconditional variance of $y_{t}^{\ddagger}$ like we obtained in $\S 3.4$ for the periodic stochastic cycle model in not available. Fortunately, $P_{*}$ is easily constructed by an extra pre-run of the Kalman filter following the ideas of Jimenez et al. (1989). Alternative solutions for $P^{*}$ from the literature on periodic ARMA models, see e.g. Lund \& Basawa (2000), are numerically unattractive in our case of a large $S$, because of the high dimension of the state.

\section{References}

Anderson, P. \& Meerschaert, M. M. (2005), 'Parameter estimation for periodically stationary time series', Journal of Time Series Analysis 26, 489-518.

Bloomfield, P., Hurd, H. L. \& Lund, R. B. (1994), 'Periodic correlation in stratospheric ozone data', Journal of Time Series Analysis 15, 127-150.

Brockwell, P. J. \& Davis, R. A. (1993), Time Series: Theory and Methods (2nd ed.), Springer-Verlag, New-York, USA.

Burridge, P. \& Taylor, R. (2004), 'Bootstrapping the HEGY seasonal unit root tests', Journal of Econometrics 123, 67-87.

Busetti, F. \& Harvey, A. C. (2003), 'Seasonality tests', Journal of Business \&3 Economic Statistics 21, 420436.

de Jong, P. (1991), 'The diffuse Kalman filter', Annals of Statistics 19, 1073-83.

Doornik, J. A. (1999), Object-Oriented Matrix Programming using Ox, 3rd edn, Timberlake Consultants Press, London.

Durbin, J. \& Koopman, S. J. (2001), Time Series Analysis by State Space Methods, Oxford University Press, Oxford. 
Fletcher, R. (1987), Practical Methods of Optimisation, (2nd Ed.), John Wiley, New York.

Franses, P. H. B. F., Boswijk, H. P. \& Haldrup, N. (1997), 'Multiple unit roots in periodic autoregression', Journal of Econometrics 80, 167-193.

Franses, P. H. \& Paap, R. (2004), Periodic Time Series Models, Oxford University Press, Oxford, U.K.

Gladysev, E. G. (1961), 'Periodically correlated random sequences', Soviet Mathematics 2, 385-388.

Hannan, E. J. (1955), 'A test for singularities in Sydney rainfall.', Australian Journal of Physics 8, 289297.

Harvey, A. C. (1989), Forecasting, structural time series models and the Kalman Filter, Cambridge University Press, Cambridge, UK.

Harvey, A. C. \& Koopman, S. J. (2000), 'Signal extraction and the formulation of unobserved components models', Econometrics Journal 3, 84-107.

Jimenez, C., McLeod, A. I. \& Hipel, K. W. (1989), 'Kalman filter estimation for periodic autoregressivemoving average models', Stochastic Hydrology and Hydraulics 3, 227-240.

Kim, C. J., Nelson, C. \& Piger, J. (2004), 'The less volatile U.S. economy: a Bayesian investigation of timing, breadth, and potential explanations', Journal of Business 63 Economic Statistics 22, 80-93.

Kitagawa, G. \& Gersch, W. (1996), Smoothness Priors Analysis of Time Series, Springer Verlag, New York.

Koopman, S. J. \& Harvey, A. C. (2003), 'Computing observation weights for signal extraction and filtering', Journal of Economic Dynamics \& Control 27, 1317-1333.

Koopman, S. J. \& Ooms, M. (2002), Periodic structural time series models: Estimation and forecasting with application, in Y. Kawasaki, ed., 'Proceedings of the 3rd International Symposium on Frontiers of Time Series Modeling: Modeling Seasonality and Periodicity, Institute of Statistical Mathematics, Tokyo, Japan, January 2002.', Institute of Statistical Mathematics, Tokyo, Japan, pp. 151-172.

Koopman, S. J. \& Ooms, M. (2006), 'Forecasting daily time series using periodic unobserved components time series models', Computational Statistics \& Data Analysis 51, 885-903.

Koopman, S. J., Shephard, N. \& Doornik, J. A. (1999), 'Statistical algorithms for models in state space using SsfPack 2.2', The Econometrics Journal 2, 107-160, www.ssfpack.com.

Lund, R. \& Basawa, I. (2000), 'Recursive prediction and likelihood evaluation for periodic ARMA models', Journal of Time Series Analysis 21, 75-93.

Lund, R., Shao, Q. \& Basawa, I. (2006), 'Parsimonious periodic time series modeling', Australian 83 New Zealand Journal of Statistics 48, 33-47.

McLeod, A. I. (1994), 'Diagnostic checking of periodic autoregression models with application', Journal of Time Series Analysis 15, 221-233. 
Ooms, M. \& Franses, P. H. (1997), 'On periodic correlations between estimated seasonal and nonseasonal components in German and U.S. unemployment', Journal of Business 85 Economic Statistics 15, 470-481.

Ooms, M. \& Franses, P. H. (2001), 'A seasonal periodic long memory model for monthly river flows', Environmental Modelling \& Software 16, 559-569.

Osborn, D. R. \& Smith, J. P. (1989), 'The performance of periodic autoregressive models in forecasting seasonal UK consumption', Journal of Business \& Economic Statistics 7, 117-127.

Primiceri, G. (2005), 'Time varying structural vector autoregressions and monetary policy', Review of Economic Studies 72, 821-852.

Proietti, T. (2004), 'Seasonal specific structural time series', Studies in Nonlinear Dynamics Ef Econometrics 8(2), Article 16, http://www.bepress.com/snde/vol8/iss2/art16.

Sensier, M. \& van Dijk, D. (2004), 'Testing for volatility changes in U.S. macroeconomic time series', The Review of Economics and Statistics 86, 833-839.

Sims, C. \& Zha, T. (2006), 'Were there regime switches in U.S. monetary policy?', American Economic Review 96, 54-81.

Tesfaye, Y. G., Meerschaert, M. M. \& Anderson, P. L. (2006), 'Identification of periodic autoregressive moving average models and their application to the modeling of river flows', Water Resources Research 42, W01419, doi:10.1029/2004WR003772, 2006.

Tiao, G. C. \& Grupe, M. R. (1980), 'Hidden periodic autoregressive-moving average models in time series data', Biometrika 67, 365-373.

Tripodis, Y. \& Penzer, J. (2004), Single season heteroscedasticity in time series, Technical Report 99, Department of Statistics, London School of Economics, forthcoming in Journal of Forecasting.

Warne, A. \& Vredin, A. (2006), 'Unemployment and inflation regimes', Studies in Nonlinear Dynamics \& Econometrics 10(2), Article 2, www.bepress.com/snde/vol10/iss2/art2.

Yao, Q. \& Brockwell, P. J. (2006), 'Gaussian maximum likelihood estimation for ARMA models. I. Time Series', Journal of Time Series Analysis 27, 857-875. 